\title{
THE $h$-PRINCIPLE AND THE EQUATIONS OF FLUID DYNAMICS
}

\author{
CAMILLO DE LELLIS AND LÁSZLÓ SZÉKELYHIDI JR.
}

AbStract. In this note we survey some recent results for the Euler equations in compressible and incompressible fluid dynamics. The main point of all these theorems is the surprising fact that a suitable variant of Gromov's $h$-principle holds in several cases.

\section{Contents}

1. Introduction

2. Weak solutions

2.1. Weak solutions with compact support in time 349

2.2. The Reynolds stress and subsolutions

2.3. Global existence of weak solutions 352

2.4. The coarse-grained flow and measure-valued solutions 352

3. Energy

3.1. Admissible weak solutions 355

3.2. Wild initial data 356

4. Applications to other equations 357

4.1. Compressible Euler 358

4.2. Active scalar equations $\quad 359$

4.3. Laminates and hulls 361

5. Onsager's Conjecture 363

5.1. A toy example 364

6. The Nash-Kuiper theorem and Gromov's $h$-principle 365

7. $C^{1, \alpha}$ isometric immersions 366

7.1. $h$-principle 366

7.2. Rigidity 368

8. Outlook and further problems $\quad 369$

Acknowledgments

About the authors $\quad 372$

References

Received by the editors November 14, 2011, and, in revised form, March 6, 2012.

2010 Mathematics Subject Classification. Primary 76B03; Secondary 76F02, 35Q35. 


\section{INTRODUCTION}

The starting point of this note is the Cauchy problem for the incompressible Euler equations,

$$
\left\{\begin{array}{l}
\partial_{t} v+\operatorname{div}_{x}(v \otimes v)+\nabla p=0, \\
\operatorname{div}_{x} v=0 \\
v(0, \cdot)=v_{0},
\end{array}\right.
$$

where the unknowns $v$ and $p$ are, respectively, a vector field and a scalar function defined on $\mathbb{R}^{n} \times[0, T)$. These fundamental equations were derived over 250 years ago by Euler and since then have played a major role in fluid dynamics. There are several outstanding open problems connected to (1) and an extensive literature about them (see for instance the surveys [3], [23], 42]). In three space dimensions little is known about smooth solutions apart from classical short-time existence and uniqueness (see [53, p 422]) and the celebrated blow-up criterion of Beale, Kato, and Majda [4] (further references for both results are [38, [47, 50, and also the books [55] and [56]). On the other hand weak solutions are known to be badly behaved from the point of view of Hadamard's well-posedness theory: in the groundbreaking paper 71] Scheffer proved the existence of a nontrivial weak solution compactly supported in time. Nevertheless, weak solutions have been studied for their expected relevance to the theory of turbulence (apparently the first to point this out is Onsager in 63]; see also [24], [40, 74]).

In this survey we argue that the nonuniqueness of weak solutions for the incompressible Euler equations should be viewed as a suitable variant of the $h$-principle. The original $h$-principle of Gromov pertains to various problems in differential geometry, where one expects high flexibility of the moduli space of solutions due to the underdetermined nature of the problem. It was not expected that the same principle and similar methods could be applied to problems in mathematical physics. We quote Gromov's speech at the Balzan Prize [44]:

The class of infinitesimal laws subjugated by the homotopy principle is wide, but it does not include most partial differential equations (expressing infinitesimal laws) of physics with a few exceptions in favor of this principle leading to unexpected solutions. In fact, the presence of the $h$-principle would invalidate the very idea of a physical law as it yields very limited global information effected by the infinitesimal data.

See also the introduction in the book 39].

As pointed out in the important paper [59] by Müller and Šverak, the existence theory for differential inclusions (see also [17, 18, 28, 48, 49]) has a close relation to the $h$-principle. In particular the method of convex integration, introduced by Gromov and extended by Müller and Šverak to Lipschitz mappings, provides a very powerful tool to construct solutions. In the paper [31 these tools were suitably modified and used for the first time to explain Scheffer's nonuniqueness theorem. It was also noticed immediately that this approach allows us to go way beyond the result of Scheffer. Indeed it has led to new developments for several equations in fluid dynamics. The goals of this note are to survey these results, list some open questions, and point out some new directions. The most exciting ones address the question of whether these methods might give us some better understanding of the theory of turbulence. 


\section{WEAK SOLUTIONS}

We start with a survey of the nonuniqueness theorems for weak solutions of (11). By a weak solution we mean, as usual, an $L_{l o c}^{2}$ vector field which solves the equations in the sense of distributions.

Definition 2.1. A vector field $v \in L_{l o c}^{2}\left(\mathbb{R}^{n} \times(0, T)\right)$ is a weak solution of the incompressible Euler equations if

$$
\int_{0}^{T} \int_{\mathbb{R}^{n}} \partial_{t} \varphi \cdot v+\nabla \varphi:(v \otimes v) d x d t=0
$$

for all $\varphi \in C_{c}^{\infty}\left(\mathbb{R}^{n} \times(0, T) ; \mathbb{R}^{n}\right)$ with $\operatorname{div} \varphi=0$ and

$$
\int_{0}^{T} \int_{\mathbb{R}^{n}} v \cdot \nabla \psi d x d t=0 \quad \text { for all } \psi \in C_{c}^{\infty}\left(\mathbb{R}^{n} \times(0, T)\right) .
$$

When $v_{0} \in L^{2}\left(\mathbb{R}^{n}\right)$, the vector field $v$ is a weak solution of (1) if (2) can be replaced by

$$
\int_{0}^{T} \int_{\mathbb{R}^{n}} \partial_{t} \varphi \cdot v+\nabla \varphi:(v \otimes v) d x d t+\int_{\mathbb{R}^{n}} \varphi(x, 0) \cdot v_{0}(x) d x=0
$$

for all $\varphi \in C_{c}^{\infty}\left(\mathbb{R}^{n} \times[0, T) ; \mathbb{R}^{n}\right)$ with $\operatorname{div} \varphi=0$.

2.1. Weak solutions with compact support in time. As already mentioned, the first nonuniqueness result for weak solutions of (1) is due to Scheffer in his groundbreaking paper [71]. The main theorem of 71] states the existence of a nontrivial weak solution in $L^{2}\left(\mathbb{R}^{2} \times \mathbb{R}\right)$ with compact support in space and time. Later on Shnirelman in 73 gave a different proof of the existence of a nontrivial weak solution in $L^{2}\left(\mathbb{T}^{2} \times \mathbb{R}\right)$ with compact support in time. In these constructions it is not clear whether the solution belongs to the energy space (we refer to the next section for the relevant definition). In the paper [31] we provided a relatively simple proof of the following stronger statement.

Theorem 2.2 (Nonuniqueness of weak solutions). There exist infinitely many compactly supported bounded weak solutions of the incompressible Euler equations in any space dimension. In particular there are infinitely many solutions $v \in L^{\infty} \cap L^{2}$ to (11) for $v_{0}=0$ and arbitrary $n \geq 2$.

2.2. The Reynolds stress and subsolutions. The proof in 31 is based on the notion of subsolution. In order to motivate its definition, let us recall the concept of Reynolds stress. It is generally accepted that the appearance of high-frequency oscillations in the velocity field is the main reason responsible for turbulent phenomena in incompressible flows. One related major problem is therefore to understand the dynamics of the coarse-grained, in other words macroscopically averaged, velocity field. If $\bar{v}$ denotes the macroscopically averaged velocity field, then it satisfies

$$
\begin{aligned}
& \partial_{t} \bar{v}+\operatorname{div}(\bar{v} \otimes \bar{v}+R)+\nabla \bar{p}=0, \\
& \operatorname{div} \bar{v}=0,
\end{aligned}
$$

where

$$
R=\overline{v \otimes v}-\bar{v} \otimes \bar{v} .
$$

The latter quantity is called Reynolds stress and arises because the averaging does not commute with the nonlinearity $v \otimes v$. On this formal level the precise definition of averaging plays no role, be it long-time averages, ensemble averages, or local 
space-time averages. The latter can be interpreted as taking weak limits. Indeed, weak limits of Leray solutions of the Navier-Stokes equations with vanishing viscosity have been proposed in the literature as a deterministic approach to turbulence (see [2], 3], 21], [52]).

A slightly more general version of this type of averaging follows the framework introduced by Tartar [83, 84, and DiPerna 35] in the context of conservation laws. We start by separating the linear equations from the nonlinear constitutive relations. Accordingly, we write (5) as

$$
\begin{aligned}
& \partial_{t} \bar{v}+\operatorname{div} \bar{u}+\nabla \bar{q}=0, \\
& \operatorname{div} \bar{v}=0,
\end{aligned}
$$

where $\bar{u}$ is the traceless part of $\bar{v} \otimes \bar{v}+R$. Since one can write

$$
R=\overline{(v-\bar{v}) \otimes(v-\bar{v})},
$$

it is clear that $R \geq 0$, i.e., $R$ is a symmetric positive semidefinite matrix. In terms of the coarse-grained variables $(\bar{v}, \bar{u})$ this inequality can be written as

$$
\bar{v} \otimes \bar{v}-\bar{u} \leq \frac{2}{n} \bar{e} I
$$

where $I$ is the $n \times n$ identity matrix and

$$
\bar{e}=\overline{\frac{1}{2}|v|^{2}}
$$

is the macroscopic kinetic energy density. Motivated by these calculations, we define subsolutions as follows. Since they will appear often, we introduce the notation $\mathcal{S}_{0}^{n \times n}$ for the vector space of symmetric traceless $n \times n$ matrices.

Definition 2.3 (Subsolutions). Let $\bar{e} \in L_{l o c}^{1}\left(\mathbb{R}^{n} \times(0, T)\right)$ with $\bar{e} \geq 0$. A subsolution to the incompressible Euler equations with given kinetic energy density $\bar{e}$ is a triple

$$
(v, u, q): \mathbb{R}^{n} \times(0, T) \rightarrow \mathbb{R}^{n} \times \mathcal{S}_{0}^{n \times n} \times \mathbb{R}
$$

with the following properties:

- $v \in L_{l o c}^{2}, \quad u \in L_{l o c}^{1}, \quad q$ is a distribution;

$$
\left\{\begin{array}{l}
\partial_{t} v+\operatorname{div} u+\nabla q=0, \quad \text { in the sense of distributions; } \\
\operatorname{div} v=0,
\end{array}\right.
$$

$$
v \otimes v-u \leq \frac{2}{n} \bar{e} I \quad \text { a.e. }
$$

Observe that subsolutions automatically satisfy $\frac{1}{2}|v|^{2} \leq \bar{e}$ a.e. (the inequality follows from taking the trace in (7)). If in addition we have the equality $\operatorname{sign} \frac{1}{2}|v|^{2}=$ $\bar{e}$ a.e., then the $v$ component of the subsolution is in fact a weak solution of the Euler equations. As mentioned above, in passing to weak limits (or when considering any other averaging process), the high-frequency oscillations in the velocity are responsible for the appearance of a nontrivial Reynolds stress. Equivalently stated, this phenomenon is responsible for the inequality sign in (7).

The key point in our approach to prove Theorem 2.2 is that, starting from a subsolution, an appropriate iteration process reintroduces the high-frequency oscillations. In the limit of this process one obtains weak solutions. However, since the oscillations are reintroduced in a very nonunique way, in fact this generates many solutions from the same subsolution. A brief outline of the relevant iteration 
scheme will be given in Section 4. In the next theorem we give a precise formulation of the previous discussion.

Theorem 2.4 (Subsolution criterion). Let $\bar{e} \in C\left(\mathbb{R}^{n} \times(0, T)\right)$ and $(\bar{v}, \bar{u}, \bar{q})$ be a smooth, strict subsolution, i.e.,

$$
(\bar{v}, \bar{u}, \bar{q}) \in C^{\infty}\left(\mathbb{R}^{n} \times(0, T)\right) \text { satisfies ([6) }
$$

and

$$
\bar{v} \otimes \bar{v}-\bar{u}<\frac{2}{n} \bar{e} \quad \text { on } \mathbb{R}^{n} \times(0, T) .
$$

Then there exist infinitely many weak solutions $v \in L_{l o c}^{\infty}\left(\mathbb{R}^{n} \times(0, T)\right)$ of the Euler equations such that

$$
\begin{aligned}
\frac{1}{2}|v|^{2} & =\bar{e} \\
p & =\bar{q}-\frac{2}{n} \bar{e}
\end{aligned}
$$

almost everywhere. Infinitely many among these belong to $C\left((0, T), L^{2}\right)$. If in addition

$$
\bar{v}(\cdot, t) \rightarrow v_{0}(\cdot) \text { in } L_{l o c}^{2}\left(\mathbb{R}^{n}\right) \text { as } t \rightarrow 0,
$$

then all the $v$ 's so constructed solve (1).

This theorem is Proposition 2 of 32 and from it we derive essentially all the results concerning the incompressible Euler equations which are surveyed in this paper. However, for a couple of cases we will need the following slightly stronger statement, which can be proved with the same methods (see also the Appendix of [81] for a more general statement):

Proposition 2.5. The same conclusions of Theorem 2.4 hold if (8) and (9) are replaced by the following weaker assumptions:

(1) $(\bar{v}, \bar{u}, \bar{q})$ is a continuous subsolution;

(2) for all $(x, t) \in \mathbb{R}^{n} \times(0, T)$,

$$
\frac{1}{2}|\bar{v}|^{2}(x, t)<\bar{e}(x, t) \quad \Rightarrow \quad \bar{v} \otimes \bar{v}(x, t)-\bar{u}(x, t)<\frac{2}{n} \bar{e}(x, t) ;
$$

(3) the open domain $\left\{\frac{1}{2}|\bar{v}|^{2}<\bar{e}\right\} \subset \mathbb{R}^{n} \times(0, T)$ is nonempty and the boundary of each time-slice has 0 Lebesgue measure.

Condition (2) amounts to the requirement that in the open subset of $\mathbb{R}^{n} \times(0, T)$ where $(\bar{v}, \bar{u}, \bar{q})$ is not a solution, it should actually be a strict subsolution.

Sketch of the proof of Proposition 2.5. The argument is exactly the same as that of Proposition 2 of [32]. A close inspection of that argument shows that it uses only the continuity of the subsolution. It remains therefore to show that the open set $\Omega \times(0, T)$ in Proposition 2 of [32] can be substituted with the more general $U:=\left\{\bar{v} \otimes \bar{v}-\bar{u}<\frac{2}{n} \bar{e}\right\}$. In the proof of [32] the assumption $U=\Omega \times(0, T)$ plays a role only in the construction of the grid of subsection 4.5 of 32 . In order to handle the case considered here, it suffices to use the same cubical partition of $\mathbb{R}^{n} \times \mathbb{R}$ and to perform the perturbations only on those closed cubes which are contained in $U$. 
2.3. Global existence of weak solutions. One way to utilize Theorem 2.4 is to start with $v_{0} \in L_{l o c}^{2}\left(\mathbb{R}^{n}\right)$, and construct a smooth triple $(\bar{v}, \bar{u}, \bar{q}) \in C^{\infty}\left(\mathbb{R}^{n} \times(0, T)\right)$ solving (6) with initial datum $v_{0}$ in the sense of (10). We further define

$$
e(v, u):=\frac{n}{2} \lambda_{\max }(v \otimes v-u),
$$

where $\lambda_{\max }$ denotes the largest eigenvalue. It is then obvious that $(\bar{v}, \bar{u}, \bar{q})$ is a smooth, strict subsolution for

$$
\bar{e}(x, t):=e(\bar{v}(x, t), \bar{u}(x, t))+\min \left(t, \frac{1}{t}\right) .
$$

Of course there are many ways of constructing such a subsolution, since the system (16) is underdetermined (there is no evolution equation for $\bar{u}$ !). This observation is closely related to the well known closure problem in turbulence.

By constructing a subsolution with bounded energy, Wiedemann in 85 recently obtained the following:

Corollary 2.6 (Global existence for weak solutions). Let $v_{0} \in L^{2}\left(\mathbb{T}^{n}\right)$ be a solenoidal vector field. Then there exist infinitely many global weak solutions of (1) with bounded energy, i.e., such that

$$
E(t)=\frac{1}{2} \int_{\mathbb{T}^{n}}|v(x, t)|^{2} d x
$$

is bounded. Moreover $E(t) \rightarrow 0$ as $t \rightarrow \infty$.

It should be noted, however, that for these solutions the energy $E(t)$ does not converge to $\frac{1}{2}\left\|v_{0}\right\|_{L^{2}}^{2}$ as $t \rightarrow 0$ : the energy will, in general, have an instantaneous jump. We will return to this issue in the next section.

2.4. The coarse-grained flow and measure-valued solutions. Following the idea that a subsolution $(\bar{v}, \bar{u}, \bar{q})$ represents the averaged (or coarse-grained) velocity, stress tensor, and pressure, respectively, it is natural to expect that, for any given subsolution, there is a sequence of weak solutions $v_{k}$ with $v_{k} \rightarrow \bar{v}$ in $L_{l o c}^{2}$ as $k \rightarrow \infty$. Indeed, this is a consequence of the proof of Theorem 2.4 one obtains a set of weak solutions which is dense in the space of subsolutions in the weak $L^{2}$ topology. For details see Section 4. This can be made even more precise with the concept of Young measures.

Let us recall that, given a sequence $\left\{v_{k}\right\} \subset L_{l o c}^{2}\left(\mathbb{R}^{n} \times[0, T)\right)$, there exists a subsequence (not relabeled) and a parametrized probability measure $\nu_{x, t}$ on $\mathbb{R}^{n} \times$ $[0, T)$, called the associated Young measure, such that

$$
f\left(v_{k}\right) \stackrel{*}{\rightarrow} \int_{\mathbb{R}^{n}} f d \nu_{x, t} \quad \text { in } L_{l o c}^{\infty} \quad \text { for all bounded continuous } f .
$$

Thus, Young measures record the one-point statistics of oscillations in weakly convergent sequences.

In order to capture both high-frequency oscillations as well as possible concentrations for Euler flows, DiPerna and Majda introduced the concept of measure-valued solutions in [36]. With this generalization, one can make sense of the limit (11) also for test functions $f$ which are not necessarily bounded, in particular for the stress tensor $f_{i j}(v)=v^{i} v^{j}$. Here we follow Alibert and Bouchitté [1]. For such test functions the limit (11) takes the form

$$
f\left(v_{k}\right) d x d t \stackrel{*}{\rightarrow}\left(\int_{\mathbb{R}^{n}} f d \nu_{x, t}\right) d x d t+\left(\int_{S^{n-1}} f^{\infty} d \nu_{x, t}^{\infty}\right) \lambda(d x d t)
$$


where the convergence is in the sense of measures and $f^{\infty}(\xi):=\lim _{s \rightarrow \infty} \frac{f(s \xi)}{s^{2}}$ is the $L^{2}$ recession function of $f$. The triple $\left(\nu, \lambda, \nu^{\infty}\right)$ is made of

- the oscillation measure $\nu_{x, t}$, which is a parametrized probability measure on $\mathbb{R}^{n}$;

- the concentration measure $\lambda=\lambda_{t} \otimes d t$, which is a nonnegative Radon measure on $\mathbb{R}^{n} \times(0, T)$;

- the concentration-angle measure $\nu_{x, t}^{\infty}$, which is a parametrized probability measure on $S^{n-1}$ and is defined $\lambda$-a.e.

Note that for bounded $f$ the formula in (12) reduces to that in (11), because $f^{\infty}=0$.

Definition 2.7 (Measure-valued solutions). A measure-valued solution of the incompressible Euler equations is a triple $\left(\nu_{x, t}, \lambda, \nu_{x, t}^{\infty}\right)$ such that the following two identities hold for all $\varphi \in C_{c}^{\infty}\left(\mathbb{R}^{n} \times(0, T) ; \mathbb{R}^{n}\right)$ with $\operatorname{div} \varphi=0$ and for all $\psi \in$ $C_{c}^{\infty}\left(\mathbb{R}^{n} \times(0, T)\right)$ :

$$
\begin{gathered}
\int_{0}^{T} \int_{\mathbb{R}^{n}} \partial_{t} \varphi \cdot\left\langle\xi, \nu_{x, t}\right\rangle \\
+\nabla \varphi:\left\langle\xi \otimes \xi, \nu_{x, t}\right\rangle d x d t \\
+\int_{0}^{T} \int_{\mathbb{R}^{n}} \nabla \varphi:\left\langle\theta \otimes \theta, \nu_{x, t}^{\infty}\right\rangle d \lambda=0, \\
\int_{0}^{T} \int_{\mathbb{R}^{n}}\left\langle\xi, \nu_{x, t}\right\rangle \cdot \nabla \psi d x d t=0 .
\end{gathered}
$$

For $v_{0} \in L^{2}\left(\mathbb{R}^{n}\right)$, the triple $\left(\nu_{x, t}, \lambda, \nu_{x, t}^{\infty}\right)$ is a measure-valued solution of (11) if (13) can be replaced by

$$
\begin{aligned}
& \int_{0}^{T} \int_{\mathbb{R}^{n}} \partial_{t} \varphi \cdot\left\langle\xi, \nu_{x, t}\right\rangle+\nabla \varphi:\left\langle\xi \otimes \xi, \nu_{x, t}\right\rangle d x d t \\
& \quad+\int_{0}^{T} \int_{\mathbb{R}^{n}} \nabla \varphi:\left\langle\theta \otimes \theta, \nu_{x, t}^{\infty}\right\rangle d \lambda+\int_{\mathbb{R}^{n}} \varphi(x, 0) \cdot v_{0}(x) d x=0
\end{aligned}
$$

for all $\varphi \in C_{c}^{\infty}\left(\mathbb{R}^{n} \times[0, T) ; \mathbb{R}^{n}\right)$ with $\operatorname{div} \varphi=0$.

In these formulas

$$
\bar{v}(x, t):=\left\langle\xi, \nu_{x, t}\right\rangle=\int_{\mathbb{R}^{n}} \xi d \nu_{x, t}(\xi)
$$

is the average (coarse-grained) velocity field, and similarly

$$
\begin{aligned}
\bar{u}+\frac{2}{n} \bar{e} I & :=\left\langle\xi \otimes \xi, \nu_{x, t}\right\rangle+\left\langle\theta \otimes \theta, \nu_{x, t}^{\infty}\right\rangle \lambda \\
& =\int_{\mathbb{R}^{n}} \xi \otimes \xi d \nu_{x, t}(\xi)+\left(\int_{S^{n-1}} \theta \otimes \theta d \nu_{x, t}^{\infty}(\theta)\right) \lambda
\end{aligned}
$$

is the average stress tensor (recall from subsection 2.2 that by $\bar{u}$ we denote the traceless part of the stress tensor). Note that in general the latter is a measure rather than a locally integrable function, because of possible $L^{2}$ concentrations. In 36. DiPerna and Majda showed that any sequence of Leray solutions of the Navier-Stokes equations converges in the vanishing viscosity limit to a measurevalued solution.

Measure-valued solutions give rise to subsolutions as in Definition 2.3. Conversely, given a subsolution $(\bar{v}, \bar{u}, \bar{q})$, it is trivial to extend it to a measure-valued solution by finding for a.e. $(x, t)$ probability measures $\nu_{x, t}$ such that (16) and (17) 
are satisfied. In this regard it is important to note that in the definition of measurevalued solutions there are no microscopic constraints, that is, constraints on the distributions of the probability measures. This is very different from other contexts where Young measures have been used, such as conservation laws in one space dimension [35, 84]: in these and similar references the Young measures satisfy additional microscopic constraints in the form of commutativity relations (for instance as a consequence of the div-curl lemma applied to the generating sequence).

Measure-valued solutions are a very weak notion, with a huge scope for unnatural nonuniqueness. However, the stronger notion of weak solution in Definition 2.1 actually exhibits this very same nonuniqueness, as witnessed by the following theorem, proved in 82 .

Theorem 2.8 (Weak solutions as 1-point statistics). Given a measure-valued solution $\left(\nu_{x, t}, \lambda, \nu_{x, t}^{\infty}\right)$ of the incompressible Euler equations, there exists a sequence of weak solutions $v_{k}$ with bounded energy such that (12) holds.

\section{ENERGY}

In the previous section we have seen that weak solutions of the Euler equations are in general highly nonunique, at least if we interpret weak solutions in the usual distributional sense of Definition 2.1. In particular the kinetic energy density $\frac{1}{2}|v|^{2}$ can be prescribed as an independent quantity. It is therefore quite remarkable that, despite this high flexibility, the additional requirement that the energy $E(t)=$ $\frac{1}{2} \int_{\mathbb{R}^{n}}|v|^{2} d x$ be nonincreasing already suffices to single out the unique classical solution when it exists.

Theorem 3.1 (Weak-strong uniqueness). Let $v \in L^{\infty}\left([0, T), L^{2}\left(\mathbb{R}^{n}\right)\right)$ be a weak solution of (11) with the additional property that $\nabla v+\nabla v^{T} \in L^{\infty}$. Assume that $\left(\nu, \lambda, \nu^{\infty}\right)$ is a measure-valued solution of (11) satisfying

$$
\int_{\mathbb{R}^{n}} \int_{\mathbb{R}^{n}}|\xi|^{2} d \nu_{x, t}(\xi) d x+\int_{\mathbb{R}^{n}} d \lambda_{t}(x) \leq \int_{\mathbb{R}^{n}}\left|v_{0}\right|^{2}(x) d x \quad \text { for a.e. } t .
$$

Then $\left(\nu, \lambda, \nu^{\infty}\right)$ coincides with $v$ as long as the latter exists, i.e.,

$$
\nu_{x, t}=\delta_{v(x, t)} \text { for a.a. }(x, t) \in \mathbb{R}^{n} \times(0, T) \text { and } \lambda \equiv 0 \text { on } \mathbb{R}^{n} \times(0, T) .
$$

This theorem recently appeared in [15, building upon ideas of [14, 16], where the authors dealt with the energy of measure-valued solutions to the Vlasov-Poisson system. More precisely, the proof of [15] yields the following information: if $\nu_{x, t}$ satisfies (18), then

$$
\bar{v}(x, t):=\int_{\mathbb{R}^{n}} \xi d \nu_{x, t}(\xi) \quad\left(=\left\langle\xi, \nu_{x, t}\right\rangle\right)
$$

is a dissipative solution of (10) in the sense of Lions (see [54]). In fact, Lions introduced the latter notion to gain back the weak-strong uniqueness while retaining the weak compactness properties of the DiPerna-Majda solutions. Theorem 3.1 shows that this can be achieved in the framework of DiPerna and Majda by simply adding the natural energy constraint (18). 
3.1. Admissible weak solutions. It is easy to see that $C^{1}$ solutions of the incompressible Euler equations satisfy the following identity, which expresses the conservation of the kinetic energy in a local form:

$$
\partial_{t} \frac{|v|^{2}}{2}+\operatorname{div}\left(\left(\frac{|v|^{2}}{2}+p\right) v\right)=0
$$

Integrating (19) in space we formally get the conservation of the total kinetic energy

$$
\frac{d}{d t} \int_{\mathbb{R}^{n}} \frac{|v|^{2}}{2}(x, t) d x=0 .
$$

These identities suggest that the notion of weak solution to (1) can be complemented with several admissibility criteria, which we list here:

(a)

$$
\int|v|^{2}(x, t) d x \leq \int\left|v_{0}\right|^{2}(x) d x \quad \text { for a.e. } t
$$

(b)

$$
\int|v|^{2}(x, t) d x \leq \int|v|^{2}(x, s) d x \quad \text { for a.e. } t>s .
$$

(c) If in addition $v \in L_{l o c}^{3}$, then

$$
\partial_{t} \frac{|v|^{2}}{2}+\operatorname{div}\left(\left(\frac{|v|^{2}}{2}+p\right) v\right) \leq 0
$$

in the sense of distributions (note that, since $-\Delta p=\operatorname{div} \operatorname{div}(v \otimes v)$, the product $p v$ is well defined by the Calderon-Zygmund inequality).

Condition (c) was proposed for the first time by Duchon and Robert in [37] and it resembles the admissibility criteria which are popular in the literature on hyperbolic conservation laws.

Next, denote by $L_{w}^{2}\left(\mathbb{R}^{n}\right)$ the space $L^{2}\left(\mathbb{R}^{n}\right)$ endowed with the weak topology. We recall that any weak solution of (11) can be modified on a set of measure zero so to get $v \in C\left([0, T), L_{w}^{2}\left(\mathbb{R}^{n}\right)\right)$ (this is a common feature of evolution equations in conservation form; see for instance Theorem 4.1.1 of [30]). Consequently, $v$ has a well-defined trace at every time, and the requirements (a) and (b) can therefore be strengthened in the following sense:

(a')

$$
\int|v|^{2}(x, t) d x \leq \int\left|v_{0}\right|^{2}(x) \mid d x \quad \text { for every } t
$$

$$
\int|v|^{2}(x, t) d x \leq \int|v|^{2}(x, s) \mid d x \quad \text { for every } t>s .
$$

However, none of these criteria restore the uniqueness of weak solutions.

Theorem 3.2 (Nonuniqueness of admissible weak solutions). Let $n \geq 2$. There exist initial data $v_{0} \in L^{\infty} \cap L^{2}$ for which there are infinitely many bounded solutions of (11) which are strongly $L^{2}$-continuous (i.e., $v \in C\left([0, \infty), L^{2}\left(\mathbb{R}^{n}\right)\right)$ ) and satisfy $\left(a^{\prime}\right),\left(b^{\prime}\right)$ and $(c)$.

The conditions $\left(a^{\prime}\right),\left(b^{\prime}\right)$ and $(c)$ hold with the equality sign for infinitely many of these solutions, whereas for infinitely many others they hold as strict inequalities. 
This theorem is from [32. The second statement generalizes the intricate construction of Shnirelman in [74, which produced the first example of a weak solution in $\mathbb{R}^{3} \times[0, \infty[$ of (1) with strict inequalities in (a) and (b).

3.2. Wild initial data. The initial data $v_{0}$ as in Theorem 3.2 are obviously not regular, since for regular initial data the local existence theorems and the weakstrong uniqueness (Theorem 3.1) ensure local uniqueness under the very mild condition (a). One might therefore ask how large is the set of these "wild" initial data. A consequence of our methods is the following density theorem (cf. with Theorem 2 in [82]).

Theorem 3.3 (Density of wild initial data). The set of initial data $v_{0}$ for which the conclusions of Theorem 3.2 holds is dense in the space of $L^{2}$ solenoidal vector fields.

Another surprising corollary is that the usual shear flow is a wild initial data. More precisely, consider the following solenoidal vector field in $\mathbb{R}^{2}$ :

$$
v_{0}(x)= \begin{cases}(1,0) & \text { if } x_{2}>0 \\ (-1,0) & \text { if } x_{2}<0\end{cases}
$$

or the following solenoidal vector field in $\mathbb{T}^{2}=\mathbb{S}^{1} \times \mathbb{S}^{1}$

$$
v_{0}(x)= \begin{cases}(1,0) & \text { if } \theta_{2} \in(-\pi, 0) \\ (-1,0) & \text { if } \theta_{2} \in(0, \pi)\end{cases}
$$

Theorem 3.4 (The vortex-sheet is wild). For $v_{0}$ as in (21) there are infinitely many weak solutions of (11) on $\mathbb{R}^{2} \times[0, \infty)$ which satisfy (c). For $v_{0}$ as in (22) there are infinitely many weak solutions of (1) on $\mathbb{T}^{2} \times[0, \infty)$ which satisfy $(c)$, $\left(a^{\prime}\right)$ and $\left(b^{\prime}\right)$.

Theorem 3.4 is proved in [80] using Proposition 2.5] and hence the proof essentially amounts to showing the existence of a suitable subsolution. See also [81] and Theorem 4.7 below for another example.

Since the various additional requirements discussed above do not ensure uniqueness of the solution even for this simple natural initial condition, Theorem 3.4 raises the following natural question: Is there a way to single out a unique, physically relevant solution? In two space dimensions one could further impose that the vorticity is a measure (à la Delort, see [34]), leading to the well-known problem of uniqueness for the vortex sheet (we note in passing that our methods do not seem to apply to the vorticity formulation of two-dimensional Euler: compare with the discussions in Section 4.2 and at the end of Section 8). Two other popular criteria considered in the literature for hyperbolic conservation laws are

(1) the vanishing viscosity limit,

(2) the maximally dissipative solution.

For scalar conservation laws they both single out the unique entropy solution (see the pioneering work of [29, where the maximal dissipation criterion was first considered; cf. also with [5]). For the two-dimensional incompressible Euler system the situation is surely more complicated. As it happens with Theorem 3.2 , some of the solutions constructed in [80] preserve the energy, whereas some others are dissipative. On the other hand, it is easy to see that the vanishing viscosity limit for initial data as in (21) or (22) is the stationary solution, which is obviously conservative. 
Therefore, even if the two criteria singled out unique weak solutions, they would be two different ones.

In [80] it is also shown that, for the two-dimensional torus $\mathbb{T}^{2}$, the maximal dissipation rate reachable with the proof of Theorem 3.4 is

$$
\max \frac{d E}{d t}=-\frac{1}{6} \text {. }
$$

It is, however, not clear whether there is a solution with this precise dissipation rate and, if it exists, whether it is unique.

\section{Applications to other EQUations}

The ideas introduced in the previous sections apply to many other nondissipative systems of evolutionary partial differential equations. We start with some general considerations and refer the reader to [49, 60] for more detail on this general framework. form

Following Tartar 84, we consider general systems in a domain $\mathcal{D} \subset \mathbb{R}^{d}$ of the

$$
\begin{aligned}
\sum_{i=1}^{d} A_{i} \partial_{i} z=0 & \text { in } \mathcal{D}, \\
z(y) \in K & \text { a.e. } y \in \mathcal{D},
\end{aligned}
$$

where

- $z: \mathcal{D} \subset \mathbb{R}^{d} \rightarrow \mathbb{R}^{N}$ is the unknown state variable,

- $A_{i}$ are constant $m \times N$ matrices,

- and $K \subset \mathbb{R}^{N}$ is a compact set

(more generally unbounded closed sets can be considered, see for instance [?]). Plane waves are solutions of (23) of the form

$$
z(x)=a h(x \cdot \xi),
$$

with $h: \mathbb{R} \rightarrow \mathbb{R}$. The wave cone $\Lambda$ is given by the states $a \in \mathbb{R}^{N}$ such that the function (25) solves (23) for any choice of the profile $h$, that is,

$$
\Lambda:=\left\{a \in \mathbb{R}^{N}: \exists \xi \in \mathbb{R}^{d} \backslash\{0\} \quad \text { with } \quad \sum_{i=1}^{d} \xi_{i} A_{i} a=0\right\} .
$$

The oscillatory behavior of solutions to the nonlinear problem is then determined by the compatibility of the set $K$ with the cone $\Lambda$. This compatibility is expressed in terms of a suitable concept of $\Lambda$-convex hull $K^{\Lambda}$ (for the precise definition, see Section 4.3). Modulo technical details, the subsolutions from Definition 2.3 are solutions $z$ of the linear relations (23) which satisfy the relaxed condition $z \in K^{\Lambda}$.

The idea of convex integration is to reintroduce oscillations by adding suitable localized versions of (25) to the subsolutions and to recover a solution of (23)-(24) iterating this process. The upshot is that in a Baire generic sense, most solutions of the "relaxed system" are actually solutions of the original system.

There are various different forms of implementing convex integration in this general framework; see for instance [28, 48, 59, 79]. A trait common to all the approaches is that one is working in a space of subsolutions in which highly oscillatory perturbations are possible. An elegant way of formalizing this was introduced by Kirchheim in 48] (see Section 3.3 therein). We recall the main steps. 
The space of subsolutions arises from a nontrivial open set

$$
\mathcal{U} \subset K^{\Lambda}
$$

satisfying the following perturbation property (cf. for instance with Proposition 2.2 in [31] and the proof of Theorem 4.1 in the same paper).

Perturbation Property (P): There is a continuous function $\varepsilon: \mathbb{R}^{+} \rightarrow \mathbb{R}^{+}$ with $\varepsilon(0)=0$ with the following property. For every $z \in \mathcal{U}$ there is a sequence of solutions $z_{j} \in C_{c}^{\infty}\left(B_{1}\right)$ of (23) such that

- $z_{j} \stackrel{*}{\rightarrow} 0$ in $L^{\infty}\left(\mathbb{R}^{d}\right)$,

- $z+z_{j}(y) \in \mathcal{U} \quad \forall y \in \mathbb{R}^{d}$,

- $\int\left|z_{j}(y)\right|^{2} d y \geq \varepsilon(\operatorname{dist}(z, K))$.

We remark that in principle the requirement $\mathcal{U} \subset K^{\Lambda}$ could be relaxed, but there are no available examples where this is done - this is related to the question of whether one-dimensional oscillations could be replaced by higher-dimensional building blocks; see e.g. the discussion in [49] and in particular Question 4 therein.

Next, let

$$
X_{0}=\left\{z \in C_{c}^{\infty}(\mathcal{D}):(23) \text { holds and } z(y) \in \mathcal{U} \text { for all } y \in \mathcal{D}\right\},
$$

and let $X$ be the closure of $X_{0}$ in $L^{\infty}(\mathcal{D})$ with respect to the weak* topology. Assuming that $K$ is bounded, the set $X$ is bounded in $L^{\infty}$ and the weak* topology is therefore metrizable on $X$.

An easy covering argument, together with property $(\mathrm{P})$, results in the following lemma.

Lemma 4.1. There is a continuous function $\tilde{\varepsilon}: \mathbb{R}^{+} \rightarrow \mathbb{R}^{+}$with $\tilde{\varepsilon}(0)=0$ such that, for every $z \in X_{0}$ there is a sequence $z_{j} \in X_{0}$ with

$$
\int_{\mathcal{D}}\left|z_{j}-z\right|^{2} d y \geq \tilde{\varepsilon}\left(\int_{\mathcal{D}} \operatorname{dist}(z(y), K) d y\right) .
$$

Since the map $z \mapsto \int_{\mathcal{D}}|z|^{2} d y$ is a Baire- 1 function on $X$, an easy application of the Baire category theorem gives that the subset of $z \in X$ satisfying (24) is Baire-generic in $X$.

The argument just sketched yields weak solutions to (23)-(24) (assuming that $(\mathrm{P})$ holds for some $\mathcal{U}$ ) which are zero on the boundary $\partial \mathcal{D}$ in the trace sense with respect to the operator in (23). In particular these weak solutions are extendable by zero to $\mathbb{R}^{d} \supset \mathcal{D}$. In applications to evolution equations, $\mathcal{D}$ is a space-time domain, say $\mathcal{D}=\mathbb{R}^{n} \times(0, T)$, and thus this argument yields weak solutions with compact time support, as in [27, 31, 75]. For the construction of weak solutions with arbitrary initial data (in particular for the construction of admissible weak solutions, as in 32]) refinements of this argument are necessary. A more detailed exposition for such cases is presented in the Appendix of [81].

In the following we survey some examples.

4.1. Compressible Euler. As a by-product of our analysis of the incompressible Euler system, nonuniqueness theorems for admissible solutions of the so-called $p$ system were proved in [32. The system of isentropic gas dynamics in Eulerian coordinates is the oldest hyperbolic system of conservation laws and consists of 
$n+1$ equations in $n$ space dimensions. The unknowns are the density $\rho$ and the velocity $v$ of the gas. The equations are

$$
\left\{\begin{array}{l}
\partial_{t} \rho+\operatorname{div}_{x}(\rho v)=0 \\
\partial_{t}(\rho v)+\operatorname{div}_{x}(\rho v \otimes v)+\nabla[p(\rho)]=0 \\
\rho(0, \cdot)=\rho^{0} \\
v(0, \cdot)=v^{0}
\end{array}\right.
$$

(cf. (3.3.17) in [30] and Section 1.1 of [72]). The pressure $p$ is a function of $\rho$, which is determined from the constitutive thermodynamic relations of the gas in question and satisfies the assumption $p^{\prime}>0$. A typical example is $p(\rho)=k \rho^{\gamma}$, with constants $k>0$ and $\gamma>1$, which gives the constitutive relation for a polytropic gas (cf. (3.3.19) and (3.3.20) of [30]).

Bounded weak solutions of (27) are bounded functions in $\mathbb{R}^{n}$ which solve it in the sense of distributions. Admissible solutions have to satisfy an additional inequality, coming from the conservation law for the energy of the system.

Definition 4.2. A weak solution of (27) is a pair $(\rho, v) \in L^{\infty}\left(\mathbb{R}^{n} \times[0, \infty)\right)$ such that the following identities hold for every test function $\psi, \varphi \in C_{c}^{\infty}\left(\mathbb{R}^{n} \times[0, \infty)\right)$ :

$$
\begin{gathered}
\int_{0}^{\infty} \int_{\mathbb{R}^{n}}\left[\rho \partial_{t} \psi+\rho v \cdot \nabla_{x} \psi\right] d x d t+\int_{\mathbb{R}^{n}} \rho^{0}(x) \psi(x, 0) d x=0 \\
\int_{0}^{\infty} \int_{\mathbb{R}^{n}}\left[\rho v \cdot \partial_{t} \varphi+\rho((v \otimes v): \nabla \varphi)\right] d x d t+\int_{\mathbb{R}^{n}} \rho^{0}(x) v^{0}(x) \cdot \varphi(x, 0) d x=0 .
\end{gathered}
$$

Consider the energy $\varepsilon: \mathbb{R}^{+} \rightarrow \mathbb{R}$ given through the law $p(r)=r^{2} \varepsilon^{\prime}(r)$. A weak solution of (27) is admissible if the following inequality holds for every nonnegative $\psi \in C_{c}^{\infty}\left(\mathbb{R}^{n} \times \mathbb{R}\right)$ :

$$
\begin{aligned}
\int_{0}^{\infty} \int_{\mathbb{R}^{n}} & {\left[\left(\rho \varepsilon(\rho)+\frac{\rho|v|^{2}}{2}\right) \partial_{t} \psi+\left(\rho \varepsilon(\rho)+\frac{\rho|v|^{2}}{2}+p(\rho)\right) v \cdot \nabla_{x} \psi\right] } \\
+ & \int_{\mathbb{R}^{n}}\left(\rho^{0} \varepsilon\left(\rho^{0}\right)+\frac{\rho^{0}\left|v^{0}\right|^{2}}{2}\right) \psi(\cdot, 0) \geq 0 .
\end{aligned}
$$

The following nonuniqueness result was proved in 32 .

Theorem 4.3 (Nonuniqueness for the $p$-system). Let $n \geq 2$. Then, for any given function $p$, there exist bounded initial data $\left(\rho^{0}, v^{0}\right)$ with $\rho^{0} \geq c>0$ for which there are infinitely many bounded admissible solutions $(\rho, v)$ of (27) with $\rho \geq c>0$.

A variant of this theorem has been recently shown by Chiodaroli using the same techniques (see [20]). Chiodaroli's Theorem highlights that the main role in this loss of uniqueness is played by the velocity field.

Theorem 4.4 (Nonuniqueness with arbitrary density). For every periodic $\rho^{0} \in C^{1}$ with $\rho^{0} \geq c>0$ there exists an initial velocity $v^{0} \in L^{\infty}$ and a time $T>0$ such that there are infinitely many bounded admissible solutions $(\rho, v)$ of (27) on $\mathbb{R}^{n} \times[0, T)$, all with density bounded away from 0.

4.2. Active scalar equations. Active scalar equations are a class of systems of evolutionary partial differential equations in $n$ space dimensions. The unknowns 
are the "active" scalar function $\theta$ and the velocity $v$, which, for simplicity, is a divergence-free vector field. The equations are

$$
\left\{\begin{array}{l}
\partial_{t} \theta+v \cdot \nabla_{x} \theta=0 \\
\operatorname{div}_{x} v=0
\end{array}\right.
$$

and $v$ and $\theta$ are coupled by an integral operator, namely

$$
v=T[\theta] \text {. }
$$

Several systems of partial differential equations in fluid dynamics fall into this class.

We rewrite (31) and (32), in the spirit of Section 2.2 (see also (6) and (7)), as the system of linear relations

$$
\left\{\begin{array}{l}
\partial_{t} \theta+\operatorname{div}_{x} q=0 \\
\operatorname{div}_{x} v=0 \\
v=T[\theta]
\end{array}\right.
$$

coupled with the nonlinear constraint

$$
q=\theta v .
$$

The initial value problem for the system (33)-(34) amounts to prescribing $\theta(x, 0)=$ $\theta_{0}(x)$.

As described at the beginning of this section, a key point is that the linear relations (33) admit a large set of plane wave solutions. Note that these linear relations are not strictly speaking of the form (23), and in order to define a suitable analog of the plane waves in this setting, we assume that the linear operator $T$ is translation invariant. Let $m(\xi)$ be its corresponding Fourier multiplier. Then we require in addition that

$$
m(\xi) \text { is } 0 \text {-homogeneous }
$$

so that (33) has the same scaling invariance as (23). Furthermore the constraint $\operatorname{div}_{x} v=0$ implies that

$$
\xi \cdot m(\xi)=0 .
$$

An important remark at this point is that the 0-homogeneity of $m$ excludes the vorticity formulation of the two-dimensional incompressible Euler equations: indeed convex integration does not seem to apply to this situation because the highest order derivatives, namely the vorticity itself, appear linearly in the equation. In a geometric context this issue has been raised by Gromov in Section 2.4.12 of 43 ] and to a certain extent analyzed in [78. Instead, the 0-homogeneity ensures that $\theta$ and $v$ are of the same order.

In spite of this restriction, several interesting equations fall into this category. Perhaps the best known examples are the surface quasi geostrophic (first proposed in 25] and usually abbreviated as SQG) and the incompressible porous medium equations (briefly IPM), corresponding, respectively, to

$$
\begin{aligned}
& m(\xi)=i|\xi|^{-1}\left(-\xi_{2}, \xi_{1}\right) \text { and } \\
& m(\xi)=|\xi|^{-2}\left(\xi_{1} \xi_{2},-\xi_{1}^{2}\right) .
\end{aligned}
$$

In 27. Cordoba, Faraco, and Gancedo proved

Theorem 4.5. Assume $m$ is given by (38). Then there exist infinitely many weak solutions of (33) and (34) in $L^{\infty}\left(\mathbb{T}^{2} \times[0,+\infty)\right)$ with $\theta_{0}=0$. 
This was generalized by Shvydkoy in [75] to all even $m(\xi)$ satisfying a mild additional regularity assumption, namely

Theorem 4.6. Assume $m$ is even, 0-homogeneous, and the set

$$
\left\{m(\xi):\left.m\right|_{\mathbb{S}^{n-1}} \text { is a regular immersion around } \xi\right\}
$$

spans $\mathbb{R}^{n}$. Then there exist infinitely many weak solutions of (33) and (34) in $L^{\infty}\left(\mathbb{T}^{2} \times[0,+\infty)\right)$ with $\theta_{0}=0$.

In the case of Theorem 4.6 modifications of the general strategy outlined in the beginning of the section are necessary. As mentioned above, (23) needs to be replaced by (33). This in turn means that in general it is not possible to obtain a sequence $\left\{z_{j}\right\}$ as in the perturbation property $(\mathrm{P})$ if we insist that each function is compactly supported. It is however possible to build up such a sequence if instead of requiring that its support be compact, we require that $z_{j}$ converges uniformly to 0 in the complement of $B_{1}(0)$ (cf. with Lemma 2.1 in [75]).

4.3. Laminates and hulls. The proofs of Theorems 4.5] and 4.6] in [27, 75] use some refined tools which were developed in the theory of laminates and differential inclusions and they present some substantial differences with the proofs in [31, 32. In order to address these differences, we start by recalling some of the standard notions in the theory of differential inclusions. These notions have been developed in the particular case of gradient vector fields (i.e., when (23) is given by $\operatorname{curl} z=0$ ), and we refer the reader to the survey [49] for a more detailed exposition.

Let us return to the general framework of (23)-(24). A function $f: \mathbb{R}^{N} \rightarrow \mathbb{R}$ on the state space is said to be $\Lambda$-convex if $t \mapsto f(a+t b)$ is convex whenever $b \in \Lambda$. Laminates are probability measures $\nu$ on $\mathbb{R}^{N}$ satisfying Jensen's inequality for $\Lambda$-convex functions,

$$
f(\bar{\nu}) \leq \int_{\mathbb{R}^{N}} f d \nu
$$

where $\bar{\nu}$ is the barycenter of $\nu$. A standard example is $\nu=\lambda \delta_{a}+(1-\lambda) \delta_{b}$ for states $a, b \in \mathbb{R}^{N}$ such that $a-b \in \Lambda$. The idea is that laminates correspond to the value-distribution of (or Young measure generated by) periodic solutions of (23). The perturbation property $(\mathrm{P})$ is implied by the following requirement: for any $z \in \mathcal{U}$, there is a laminate $\nu$ such that

$$
\begin{aligned}
& \text { - } \bar{\nu}=z \\
& \text { - } \operatorname{supp} \nu \subset \mathcal{U}, \\
& \text { - } \int|\zeta-z|^{2} d \nu(\zeta) \geq \varepsilon(\operatorname{dist}(z, K)) \text {. }
\end{aligned}
$$

The passage from this property to $(\mathrm{P})$ is nontrivial and requires localization arguments. Nevertheless, assuming that this can be done, a natural candidate for $\mathcal{U}$ is the interior of the $\Lambda$-convex hull, defined by

$$
K^{\Lambda}=\{\bar{\nu}: \nu \text { is a laminate with } \operatorname{supp} \nu \subset K\} .
$$

Thus, a large $\Lambda$-convex hull signifies a large set of oscillatory solutions of (23) compatible with (24).

In [27] and [75] the authors avoid calculating the full hull and instead restrict themselves to exhibiting a nontrivial (but possibly much smaller) open set $\mathcal{U}$ satisfying $(\mathrm{P})$. However, in exchange they are forced to use much more complicated sequences $z_{j}$ (corresponding to more complicated laminates). Indeed, the $z_{j}$ 's used in [31] are localizations of simple plane waves, whereas the ones used in [27] and 
[75] arise as an infinite nested sequence of repeated plane waves. The obvious advantages of the latter approach are its robustness and generality. This is useful in cases where an explicit computation of the hull $K^{\Lambda}$ (or even of the convex hull!) is out of reach due to the high complexity and high dimensionality.

On the other hand, there are advantages in computing the hull $K^{\Lambda}$. First of all, in many cases $K^{\Lambda}$ can be characterized by separation arguments as

$$
K^{\Lambda}=\left\{a: f(a) \leq \sup _{K} f \text { for all } \Lambda \text {-convex } f\right\} .
$$

This is the case when (23) is given by $\operatorname{curl} z=0$; see [48, 66]. The arguments can quite probably be generalized under mild assumptions on $\Lambda$, although to our knowledge this has not been done. However, for our purposes in this article it suffices to take this equivalence as a guiding principle. For instance, in Theorem 2.4 condition (9) characterizes precisely the interior of $K^{\Lambda}$, which in this case is the interior of the convex hull $K^{c o}$.

Secondly, computing the hull $K^{\Lambda}$ gives optimal criteria for wild initial data, as in Section 3.2. Indeed, observe that in Theorem 2.4, the initial data $v_{0}$ is given by the subsolution through (10). In the case of Theorem 4.5, that is, for the incompressible porous medium equation, the set $K^{\Lambda}$ was computed in [81. As a consequence one obtains the following existence theorem for the Muskat problem (see Theorem 1.1 in [81]).

Theorem 4.7. Assume $m$ is given by (38). Then there exist infinitely many weak solutions of (33) and (34) in $L^{\infty}\left(\mathbb{R}^{2} \times[0,+\infty)\right)$ with $|\theta|=1$ a.e. and

$$
\theta_{0}(x)= \begin{cases}1 & \text { if } x_{2}>0 \\ -1 & \text { if } x_{2}<0\end{cases}
$$

In fact the solutions constructed in this theorem exhibit a mixing zone (i.e., where $\theta$ oscillates wildly between \pm 1 ) around the initial interface $\left\{x_{2}=0\right\}$, which expands linearly in time.

We recall that the coarse-grained flow for this problem has been analyzed in detail in 64, 65. In 64. Otto introduced a relaxation approach for the incompressible porous media equation, based on the gradient flow formulation and using ideas from mass transport. It was shown that, under certain assumptions, there exists a unique "relaxed" solution $\bar{\theta}$, representing a kind of coarse-grained density. Moreover, Otto proved that, in general, the mixing zone (where the coarse-grained density $\bar{\theta}$ is strictly between \pm 1 ) grows linearly in time, with the possible exception of a small set of volume fraction $O\left(t^{-1 / 2}\right)$ (cf. with [65, Remark 2.1]). Following the general considerations in Section 2.2, we can interpret $\bar{\theta}$ as the subsolution corresponding to the solutions in Theorem 4.7, for which there exists a sequence $\theta_{k}$ of weak solutions such that $\theta_{k} \stackrel{*}{\rightarrow} \bar{\theta}$.

Although subsolutions are clearly not unique, the coarse-grained density of Otto turns out to be extremal in the sense that it corresponds to the maximal expansion of the mixing zone. It is therefore interesting to note that, although weak solutions are clearly not unique, there is a way to identify a selection criterion among subsolutions which leads to uniqueness. 


\section{Onsager's Conjecture}

The relevance of weak solutions of the Euler equations to turbulence has long been surmised. One key question is related to the phenomenon of anomalous dissipation. This experimentally observed fact, namely that the rate of energy dissipation in the vanishing viscosity limit stays above a certain nonzero constant, is expected to arise from a mechanism of transporting energy from large to small scales (known as an energy cascade) via the nonlinear transport term in the NavierStokes equations, rather than the (dissipative) viscosity term. Motivated by this idea, Onsager conjectured in the 1940s that there exist weak solutions of the Euler equations which dissipate energy. More precisely, the conjecture was that there exist dissipative weak solutions in the Hölder space $C^{\alpha}$ with $\alpha<1 / 3$, whereas if $\alpha>1 / 3$, then the energy is conserved (see [63] and also [4]).

The latter statement has been rigorously proved with various sharper versions in [24, 40, 37]; in particular we refer the reader to the recent survey [76].

On the other hand there is no known construction of a dissipative weak solution in $C^{\alpha}$ with any $\alpha \geq 0$ (except for the very recent paper [33]). As pointed out in Theorem 3.2 , one can use Theorem 2.4 to produce dissipative solutions which are merely $L^{\infty}$, and prior to that the only known construction of a weak solution with dissipation, due to Shnirelman [74, produced an $L^{2}$ solution. Despite this, it is instructive to take a second look at the construction in Theorem 2.4 in light of expectations regarding the energy spectrum and the conjecture of Onsager.

In Section 4 we presented the so-called Baire category method, which is in some sense not constructive. However, the same idea of adding oscillatory perturbations can be implemented in a constructive way as well; see for instance Section 5 of [31]. In a nutshell the idea is to define a sequence of subsolutions $\left(v_{k}, u_{k}, q_{k}\right)$ as

$$
\left(v_{k+1}, u_{k+1}, q_{k+1}\right)(x, t)=\left(v_{k}, u_{k}, q_{k}\right)(x, t)+\left(V_{k}, U_{k}, Q_{k}\right)\left(x, t, \lambda_{k} x, \lambda_{k} t\right) .
$$

The map

$$
(\xi, \tau) \mapsto\left(V_{k}, U_{k}, Q_{k}\right)(x, t, \xi, \tau)
$$

is a periodic plane-wave solution of (6) with average 0 , parametrized by $(x, t)$. The positive number $\lambda_{k}$ is a (large) frequency to be chosen. The aim is to choose the plane-wave $\left(V_{k}, U_{k}, Q_{k}\right)$ and the frequency $\lambda_{k}$ iteratively in such a way that

- $\left(v_{k}, u_{k}, q_{k}\right)$ continues to satisfy (6) (strictly speaking this requires an additional corrector term in the scheme (39)),

- the inequality (7) holds,

- and

$$
v_{k} \otimes v_{k}-u_{k} \rightarrow \frac{2}{n} \bar{e} I
$$

Observe that, because of inequality (7), it suffices to show the weak convergence in (40).

The role of the frequency can be explained as follows: Using (39) we expand as

$$
v_{k+1} \otimes v_{k+1}-u_{k+1}=v_{k} \otimes v_{k}-u_{k}+\left(v_{k} \otimes V_{k}+V_{k} \otimes v_{k}-U_{k}\right)+V_{k} \otimes V_{k} .
$$

For large $\lambda_{k}$ the term in brackets converges to zero weakly, whereas the term $V_{k} \otimes V_{k}$ is nonnegative. Therefore, if the sequence $\left\{\lambda_{k}\right\}_{k \in \mathbb{N}}$ converges to $\infty$ sufficiently fast, then the sequence of tensors $\left\{v_{k} \otimes v_{k}-u_{k}\right\}$ is monotone, with a uniform bound given by (7). The strong convergence follows. 
Thus, convergence of this constructive scheme is improved by choosing the frequencies $\lambda_{k}$ higher and higher. In terms of the energy spectrum this amounts to eddies (the periodic perturbations) being placed in well separated frequencies where the interaction between neighboring frequencies is negligible.

On the other hand clearly any (fractional) derivative or Hölder norm of $v_{k}$ gets worse by such a choice of $\lambda_{k}$. The best regularity admitted by the scheme corresponds to the slowest rate at which the frequencies $\lambda_{k}$ tend to infinity whilst still leading to convergence.

5.1. A toy example. We will consider here a toy model, where the considerations above can be demonstrated and which has been suggested by the anonymous referee to [31. Consider the problem of exhibiting functions $u:[0,1] \rightarrow \mathbb{R}$ such that $|u|=1$. In the context of (23) $-(24)$ this corresponds to $K=\{-1,1\}$ and the differential constraint being void. The following scheme aims at producing such functions. We start with a given function $u_{0}:[0,1] \rightarrow(-1,1)$ and build a sequence with the following iteration scheme:

$$
u_{k+1}(x)=u_{k}(x)+\frac{1}{2}\left[1-u_{k}^{2}(x)\right] s\left(\lambda_{k} x\right),
$$

where $s: \mathbb{R} \rightarrow \mathbb{R}$ is the 1 -periodic extension of $\mathbf{1}_{(0,1 / 2]}-\mathbf{1}_{(1 / 2,1]}$ and $\lambda_{k}>1$ is a sequence of frequencies still to be fixed. The following assertions are straightforward:

- If $\sup _{[0,1]}\left|u_{k}\right|<1$, then also $\sup _{[0,1]}\left|u_{k+1}\right|<1$.

- If $\sup _{[0,1]}\left|u_{0}\right|<1$ and $u_{k} \rightarrow u_{\infty}$ in $L^{1}(0,1)$, then $\left|u_{\infty}\right|=1$ a.e.

Therefore, in order to produce a solution to our toy problem, it suffices to choose the sequence $\left\{\lambda_{k}\right\}$ so to ensure the strong convergence of $u_{k}$. To this end observe that

$$
\int_{0}^{1}\left|u_{k+1}\right|^{2} d x=\int_{0}^{1}\left(\left|u_{k}\right|^{2}+\frac{1}{4}\left(1-u_{k}^{2}\right)^{2}+u_{k}\left(1-u_{k}^{2}\right) s\left(\lambda_{k} x\right)\right) d x .
$$

Moreover, as $\lambda \rightarrow \infty$, we have $s(\lambda x) \rightarrow 0$ in $L^{2}(0,1)$. Therefore, by choosing $\lambda_{k}$ sufficiently large (depending on $u_{k}$ ), we can ensure that

$$
\int_{0}^{1}\left|u_{k+1}\right|^{2} d x \geq \int_{0}^{1}\left|u_{k}\right|^{2} d x+\int_{0}^{1} \frac{1}{8}\left(1-u_{k}^{2}\right)^{2} d x .
$$

The strong convergence then follows easily. Here we see that choosing $\lambda_{k}$ to be a rapidly increasing sequence "helps" the strong convergence of the scheme.

However, it is also clear that for any additional regularity of the limit $u_{\infty}$ one should choose $\lambda_{k}$ to increase as slowly as possible. More precisely, the optimal regularity that is reachable via this iteration scheme will depend on the connection between the choice of $\lambda_{k}$ with the rate of convergence of the scheme. To see this, observe that - roughly speaking - fractional Sobolev regularity of $u_{\infty}$ will follow from interpolating between the norms

$$
\begin{aligned}
\left\|u_{k+1}-u_{k}\right\|_{L^{1}} & \sim \int_{0}^{1}\left(1-u_{k}^{2}\right) d x, \\
\left\|u_{k+1}-u_{k}\right\|_{B V} & \sim \lambda_{k} \int_{0}^{1}\left(1-u_{k}^{2}\right) d x .
\end{aligned}
$$

Therefore the following statement is of interest, showing that exponential growth of the frequencies leads to exponential convergence of the scheme: 
Lemma 5.1. Let $\lambda_{k}=2^{k}$. Then

$$
\int_{0}^{1} \sqrt{1-u_{k}^{2}} d x \leq\left(\frac{7}{8}\right)^{k} \int_{0}^{1} \sqrt{1-u_{0}^{2}} d x .
$$

Proof. By the choice of the oscillatory function $s$, we see that the following identity holds for any continuous function $f \in C(-1,1)$ :

$$
\int_{0}^{1} f\left(u_{k+1}\right) d x=\int_{0}^{1} \frac{1}{2}\left[f\left(u_{k}-\frac{1}{2}\left(1-u_{k}^{2}\right)\right)+\frac{1}{2} f\left(u_{k}+\frac{1}{2}\left(1-u_{k}^{2}\right)\right)\right] d x
$$

Now set $f(u):=\left(1-u^{2}\right)^{1 / 2}$. By direct calculation, we obtain

$$
f^{\prime \prime}(u)=-\left(1-u^{2}\right)^{-3 / 2},
$$

and hence $f^{\prime \prime}(u)\left(1-u^{2}\right)^{2}=-f(u)$. Moreover, $f^{(4)} \leq 0$ on $(-1,1)$. This implies that $f^{\prime \prime}(\xi) \leq f^{\prime \prime}(u)+f^{\prime \prime \prime}(u)(\xi-u)$ for every $\xi$. Thus the function $g(\xi)=f(\xi)-$ $f^{\prime \prime}(u) \frac{(\xi-u)^{2}}{2}-f^{\prime \prime \prime}(u) \frac{(\xi-u)^{3}}{6}$ is concave. From the inequality $\frac{1}{2} g(u-v)+\frac{1}{2} g(u+v) \leq$ $g(u)$, we then conclude

$$
\frac{1}{2} f(u-v)+\frac{1}{2} f(u+v) \leq f(u)+\frac{1}{2} f^{\prime \prime}(u) v^{2} .
$$

In particular, setting $v=\frac{1}{2}\left(1-u^{2}\right)$, we obtain

$$
\frac{1}{2} f\left(u-\frac{1}{2}\left(1-u^{2}\right)\right)+\frac{1}{2} f\left(u+\frac{1}{2}\left(1-u^{2}\right)\right) \leq \frac{7}{8} f(u) .
$$

We conclude

$$
\int_{0}^{1} f\left(u_{k+1}\right) d x \leq \frac{7}{8} \int_{0}^{1} f\left(u_{k}\right) d x
$$

and the lemma follows.

\section{The NAsh-Kuiper theorem And Gromov's $h$-PRINCIPle}

The origin of convex integration lies in the famous Nash-Kuiper theorem. In this section we briefly recall some landmark results from the theory of isometric embeddings.

Let $M^{n}$ be a smooth compact manifold of dimension $n \geq 2$, equipped with a Riemannian metric $g$. An isometric immersion of $\left(M^{n}, g\right)$ into $\mathbb{R}^{m}$ is a map $u \in$ $C^{1}\left(M^{n} ; \mathbb{R}^{m}\right)$ such that the induced metric $u^{\sharp} e$ agrees with $g$. In local coordinates this amounts to the system

$$
\partial_{i} u \cdot \partial_{j} u=g_{i j}
$$

consisting of $n(n+1) / 2$ equations in $m$ unknowns. If in addition $u$ is injective, it is an isometric embedding. Assume for the moment that $g \in C^{\infty}$. The two classical theorems concerning the solvability of this system follow.

(A) If $m \geq(n+2)(n+3) / 2$, then any short embedding can be uniformly approximated by isometric embeddings of class $C^{\infty}$ (Nash [62], Gromov [43]).

(B) If $m \geq n+1$, then any short embedding can be uniformly approximated by isometric embeddings of class $C^{1}$ (Nash 61, Kuiper [51]).

Recall that a short embedding is an injective map $u: M^{n} \rightarrow \mathbb{R}^{m}$ such that the metric induced on $M$ by $u$ is shorter than $g$. In coordinates this means that

$$
\left(\partial_{i} u \cdot \partial_{j} u\right) \leq\left(g_{i j}\right)
$$


in the sense of quadratic forms. Thus, (A) and (B) are not merely existence theorems, they show that there exists a huge (essentially $C^{0}$-dense) set of solutions. This type of abundance of solutions is a central aspect of Gromov's $h$-principle, for which the isometric embedding problem is a primary example (see [39, 43]).

There is a clear formal analogy between (41)-(42) and (11), (6), and (77). First of all, note that the Reynolds stress measures the defect to being a solution of the Euler equations and it is in general a nonnegative symmetric tensor, whereas $g_{i j}-\partial_{i} u \cdot \partial_{j} u$ measures the defect to being isometric and, for a short map, is also a nonnegative symmetric tensor. More precisely (41) can be formulated for the deformation gradient $A:=D u$ as the coupling of the linear constraint

$$
\operatorname{curl} A=0
$$

with the nonlinear relation

$$
A^{t} A=g .
$$

In this sense short maps are "subsolutions" to the isometric embedding problem in the spirit of Definition 2.3. Along this line of thought, Theorem 2.4 is then the analogue for the Euler equations of the Nash-Kuiper result (B). However note that, strictly speaking, the formal analog of statement (B) would be replacing $L^{\infty}$ by $C^{0}$ in Theorem 2.4. A result in this direction has been proved only very recently in [33].

Statement (B) is rather surprising for two reasons. First of all, for $n \geq 3$ and $m=n+1$, the system (41) is overdetermined. Moreover, for $n=2$ we can compare (B) to the classical rigidity result concerning the Weyl problem: if $\left(S^{2}, g\right)$ is a compact Riemannian surface with positive Gauss curvature and $u \in C^{2}$ is an isometric immersion into $\mathbb{R}^{3}$, then $u$ is uniquely determined up to a rigid motion ([22, 45], see also [77] Chapter 12 for a thorough discussion). Thus it is clear that isometric immersions have a completely different qualitative behavior at low and high regularity (i.e., below and above $C^{2}$ ).

A strikingly similar phenomenon holds for the Euler equations since, when coupled with the energy constraint $|v|^{2}=2 \bar{e}$, they are also formally overdetermined. Moreover $C^{1}$ solutions of the Cauchy problem are unique. There are further similarities regarding the Hölder regularity, as we will show in Section 7 below.

\section{7. $C^{1, \alpha}$ ISOMETRIC IMMERSIONS}

7.1. $h$-principle. The question of a sharp regularity threshold has been the object of investigation for the isometric embedding of surfaces as well (see for instance [43, [86]). As already mentioned, the isometric embeddings of $\mathbb{S}^{2}$ into $\mathbb{R}^{3}$ are rigid in the class $C^{2}$, whereas the $h$-principle holds for $C^{1}$. Borisov investigated embeddings of class $C^{1, \alpha}$ and proved the rigidity for $\alpha>2 / 3$ ([8], 10]) and the $h$-principle for $\alpha<1 / 13$ (although the latter was announced in 1965, see [11, a partial proof only appeared in 2004 [12]). In [26] we returned to this problem, and gave a more modern PDE proof of the $h$-principle for $\alpha<1 / 7$, namely

Theorem 7.1 (Local existence). Let $n \in \mathbb{N}, g_{0} \in \operatorname{sym}_{n}^{+}$and $n_{*}=\frac{n(n+1)}{2}$. There exists $r>0$ such that the following holds for any smooth bounded open set $\Omega \subset \mathbb{R}^{n}$ and any Riemannian metric $g \in C^{\beta}(\bar{\Omega})$ with $\beta>0$ and $\left\|g-g_{0}\right\|_{C^{0}} \leq r$. There 
exists a constant $\delta_{0}>0$ such that, if $u \in C^{2}\left(\bar{\Omega} ; \mathbb{R}^{n+1}\right)$ and $\alpha$ satisfy

$$
\left\|u^{\sharp} e-g\right\|_{0} \leq \delta_{0}^{2} \quad \text { and } \quad 0<\alpha<\min \left\{\frac{1}{1+2 n_{*}}, \frac{\beta}{2}\right\},
$$

then there exists a map $v \in C^{1, \alpha}\left(\bar{\Omega} ; \mathbb{R}^{n+1}\right)$ with

$$
v^{\sharp} e=g \quad \text { and } \quad\|v-u\|_{C^{1}} \leq C\left\|u^{\sharp} e-g\right\|_{C^{0}}^{1 / 2} .
$$

Corollary 7.2 (Local $h$-principle). Let $n, g_{0}, \Omega, g, \alpha$ be as in Theorem 17.1, Given any short map $u \in C^{1}\left(\bar{\Omega} ; \mathbb{R}^{n+1}\right)$ and any $\varepsilon>0$, there exists an isometric immersion $v \in C^{1, \alpha}\left(\bar{\Omega} ; \mathbb{R}^{n+1}\right)$ with $\|u-v\|_{C^{0}} \leq \varepsilon$.

The proof of Theorem 7.1 is based on an iteration scheme which follows the method of Nash and Kuiper [51, 61]. The iteration consists of stages, and each stage consists of several steps. The purpose of a stage is to correct the error $g-u^{\sharp} e$. In order to achieve this correction, the error is decomposed into a sum of primitive metrics as

$$
g-u^{\sharp} e=\sum_{k=1}^{n_{*}} a_{k}^{2} \nu_{k} \otimes \nu_{k} .
$$

To keep the notation simpler, in what follows we will use the abbreviations $\|\cdot\|_{N}$ and $\|\cdot\|_{k, \alpha}$ for the $C^{N}$ and $C^{k, \alpha}$ norms, respectively. The natural estimates associated with (43) are

$$
\begin{aligned}
& \left\|a_{k}\right\|_{0} \sim\left\|g-u^{\sharp} e\right\|_{0}^{1 / 2}, \\
& \left\|a_{k}\right\|_{N+1} \sim\|u\|_{N+2} \quad \text { for } N=0,1,2, \ldots .
\end{aligned}
$$

A step then involves adding one primitive metric. In other words the goal of a step is the metric change

$$
u^{\sharp} e \mapsto u^{\sharp} e+a^{2} \nu \otimes \nu .
$$

Nash used spiraling perturbations (also known as the "Nash twist") to achieve this; in codimension 1, Kuiper replaced the spirals by corrugations: in both cases the new map $\tilde{u}$ is of the form

$$
\tilde{u}(x)=u(x)+\frac{1}{\lambda} w(x, \lambda x \cdot \nu)
$$

for some appropriate choice of $w$, where $w$ is periodic in the second variable. The parameter $\lambda$ is given by

$$
\lambda=K \frac{\|u\|_{2}}{\sqrt{\left\|g-u^{\sharp} e\right\|_{0}}},
$$

where $K$ is a large (but fixed) constant. One checks that the addition of a primitive metric is then possible with the estimates

$$
\begin{aligned}
C^{0} \text {-error in the metric } & \sim\left\|g-u^{\sharp} e\right\|_{0} \frac{1}{K}, \\
\text { increase of } C^{1} \text {-norm of } u & \sim\left\|g-u^{\sharp} e\right\|_{0}^{1 / 2}, \\
\text { increase of } C^{2} \text {-norm of } u & \sim\|u\|_{2} K
\end{aligned}
$$

for any $K \geq 1$. Observe that the first two of these estimates are essentially the same as in [51, 61. Furthermore, the third estimate is only valid modulo a "loss of derivative". The exponent $1 / 2$ in the second estimate is due to the quadratic nature of the nonlinearity. 
The low codimension forces the steps to be performed serially. Thus the number of steps in a stage equals the number of primitive metrics in the above decomposition which interact. This is given by $n_{*}=n(n+1) / 2$. To deal with the "loss of derivative" problem in [26] we mollify the map $u$ at the start of every stage, in a similar manner as is done in a Nash-Moser iteration (see [57, 58]). A careful estimate (using Proposition 7.3) shows that this can be done without any additional error. Therefore, iterating the estimates for one step over a single stage (that is, over $n_{*}$ steps) leads to

$$
\begin{aligned}
C^{0} \text {-error in the metric } & \sim\left\|g-u^{\sharp} e\right\|_{0} \frac{1}{K}, \\
\text { increase of } C^{1} \text {-norm of } u & \sim\left\|g-u^{\sharp} e\right\|_{0}^{1 / 2}, \\
\text { increase of } C^{2} \text {-norm of } u & \sim\|u\|_{2} K^{n_{*}} .
\end{aligned}
$$

With these estimates, iterating over the stages leads to exponential convergence of the metric error, to a controlled growth of the $C^{1}$ norm and to an exponential growth of the $C^{2}$ norm of the map. In particular, interpolation between these two norms leads to convergence in $C^{1, \alpha}$ for $\alpha<\frac{1}{1+2 n_{*}}=\frac{1}{1+n(n+1)}$.

Recalling the analogy with the Euler equations, which amounts to identifying the velocity $v$ with the deformation gradient $D u$, the analogous scheme for producing $C^{0, \alpha}$ solutions of Euler would involve perturbations of the form

$$
\tilde{v}(x, t)=v(x, t)+w(x, t, \lambda x, \lambda t)
$$

(with $w$ periodic and average 0 in the second variable). If the estimates analogous to (44)-(46) would carry over with one single step in a stage (i.e., $n_{*}=1$ ), one would reach the exponent conjectured by Onsager. This Ansatz for the critical exponent $1 / 3$ is independent of considerations on the energy spectrum, giving an entirely new point of view on the famous Kolmogorov 5/3 law.

7.2. Rigidity. We turn next to the Weyl problem, i.e., the characterization of isometric embeddings of two-dimensional positively curved surfaces in $\mathbb{R}^{3}$. Concerning the rigidity, it is known from the work of Pogorelov and Sabitov that

(1) closed $C^{1}$ surfaces with positive Gauss curvature and bounded extrinsic curvature are convex (see [67]);

(2) closed convex surfaces are rigid in the sense that isometric immersions are unique up to rigid motion 68 ;

(3) a convex surface with metric $g \in C^{l, \beta}$ with $l \geq 2,0<\beta<1$ and positive curvature is of class $C^{l, \beta}$ (see [67, 70]).

Thus, extending the rigidity in the Weyl problem to $C^{1, \alpha}$ isometric immersions can be reduced to showing that the image of the surface has bounded extrinsic curvature in the sense of Pogorelov. This concept is easily explained in the following way. Let $(M, g)$ be a smooth two-dimensional closed Riemannian manifold, and let $u: M \rightarrow \mathbb{R}^{3}$ be an isometric immersion. According to Gauss's original definition, the Gauss curvature of $u(M)$ can be defined as the area distortion of the Gauss map $N$. In a weak sense, this is a well-defined object even when the immersion is merely a $C^{1}$ map, because in this case $N$ is continuous. The immersed surface $u(M)$ is then said to have bounded extrinsic curvature if this area distortion is bounded.

Using geometric arguments, in the series of papers 6, 7, 8, 9, 10, Borisov proved that for $\alpha>2 / 3$ the image of surfaces with positive Gauss curvature has indeed bounded extrinsic curvature. Consequently, rigidity holds in this range and in 
particular $2 / 3$ is an upper bound on the range of Hölder exponents that allow for the $h$-principle.

A short proof of Borisov's rigidity result was provided in [26]. Note that if $u \in C^{3}$, one can compute the area distorsion of the Gauss map from the Riemanncurvature tensor, which in turn depends only on the metric; we call this scalar field $\kappa$. Even if the metric $g$ is smooth, nonetheless this identity is in general false if the isometry is not regular enough, as shown precisely by the Nash-Kuiper theorem. However, in 26 we showed that the $C^{1, \frac{2}{3}+\varepsilon}$ regularity is enough for this identity to hold. A key observation is that this identity can be expressed in the following integral formula

$$
\int_{V} f(N(x)) \kappa(x) d A(x)=\int_{\mathcal{S}^{2}} f(y) \operatorname{deg}(y, V, N) d \sigma(y),
$$

where

- $V$ is an arbitrary open subset of $M$,

- $f$ is any $C^{1}$ function on $\mathbb{S}^{2}$,

- $\operatorname{deg}(y, V, N)$ is the Brouwer degree of the map $\left.N\right|_{V}$ at $y$,

- $d A$ denotes the Riemannian volume form on $(M, g)$,

- $d \sigma$ is the standard surface measure on $\mathbb{S}^{2}$.

The identity (47) is just a change of variable formula and can be proved for isometric immersions $u \in C^{1,2 / 3+\varepsilon}$ by a regularization argument. The proof in 26 relies on commutator estimates (see Proposition 7.3 below), which are surprisingly close to the argument of [24] for proving the energy conservation of $C^{0, \alpha}$ solutions of Euler when $\alpha>1 / 3$.

Proposition 7.3 (Quadratic estimate). Let $\Omega \subset \mathbb{R}^{n}$ be an open set, $v \in C^{1, \alpha}\left(\Omega, \mathbb{R}^{m}\right)$, and let $e$ be the Euclidean metric on $\mathbb{R}^{m}$. Consider the corresponding pull-back metric $\left(v^{\sharp} e\right)_{i j}=\partial_{i} v \cdot \partial_{j} v$. Assume $v^{\sharp} e \in C^{2}$, and let $\varphi \in C_{c}^{\infty}\left(\mathbb{R}^{n}\right)$ be a standard symmetric convolution kernel. Then, for every compact set $K \subset \Omega$,

$$
\left\|\left(v * \varphi_{\ell}\right)^{\sharp} e-v^{\sharp} e\right\|_{C^{1}(K)}=O\left(\ell^{2 \alpha-1}\right) .
$$

In the case of isometric embeddings there does not seem to be a universally accepted critical exponent (see Problem 27 in [86]), even though $1 / 2$ and $1 / 3$ both seem relevant (compare with the discussion in [12]). In particular, fix a map $u$ and a kernel $\varphi$ satisfying the assumptions of the proposition with $\alpha>1 / 2$. Then the Christoffel symbols of $\left(v * \varphi_{\ell}\right)^{\sharp} e$ converge uniformly to those of $v^{\sharp} e$. This corresponds to the results of Borisov in [6, 7] and hints at the absence of $h$-principle for $C^{1, \frac{1}{2}+\varepsilon}$ immersions. One might further notice that the regularity $C^{1, \frac{1}{3}+\varepsilon}$ is still enough to guarantee a very weak notion of convergence of the Christoffel symbols.

\section{OUTLOOK AND FURTHER PROBLEMS}

We list in this section several open questions which remain to be addressed.

Problem 1. Analogues of Theorem 3.4 for compressible Euler.

Different behaviors might be expected for different types of Riemann problems. The direct analogue of Theorem 3.4 would be a contact discontinuity, and it seems 
likely that some form of the statement survives for compressible Euler. Nevertheless, because of the different role of the pressure term, a direct transfer from the incompressible to the compressible case (as was done for Theorem 4.3 in [32]) seems impossible.

Problem 2. Compute the hull for compressible Euler.

The proof of Theorem 4.3 essentially relies on the observation that Theorem 2.4 gives weak solutions of the incompressible Euler system (1) with fixed $|v|^{2}$ and fixed pressure $p$. Thus, an appropriate choice automatically yields weak solutions of the compressible Euler system (27). Within the general framework of (23)-(24) this route via the incompressible Euler equations amounts to restricting to a smaller constitutive set $K$ for compressible Euler, where $\rho$ is fixed to be constant. A further step was obtained recently by Chiodaroli (cf. Theorem 4.4 above), but the general case, which would allow for wild oscillations in the density as well as the velocity, remains open. In other words, one should define the state space and the constitutive set for compressible Euler in full generality, and compute the hull. This would lead to the correct definition of subsolution, and thus eventually to the solution of the next problem:

Problem 3. Analogue of Theorem 2.8 for compressible Euler.

As noted in Section 7.2, although there is a surprising number of similarities between weak solutions of the Euler equations and the Nash-Kuiper theorem, in contrast to Euler there are no natural conjectures for critical Hölder exponents for isometric embeddings. Therefore, a central question is:

Problem 4. Find the optimal Hölder exponent for Theorem 7.1 and Corollary 7.2 ,

It should be pointed out that for incompressible Euler the exponent $1 / 3$ is conjectured to be critical with respect to the energy conservation. It is not known whether being above this exponent implies uniqueness. On the other hand the rigidity statement in Section 7.2 is a uniqueness statement. A conceivable scenario is then the existence of other regimes between the exponents $1 / 3$ and $2 / 3$ for isometric embeddings, where the rigidity does not hold but the $h$-principle is also excluded.

In Section 7 we restricted our attention to isometric embeddings in codimension 1. Looking closely at the construction it is natural to expect that higher codimension for the embedding should result in better regularity. Indeed, for the case of sufficiently large codimension, A. Källen showed in [46] that the regularity in Theorem 7.1 and Corollary 7.2 can be pushed up to any $\alpha<1$, provided the metric is sufficiently smooth!

Problem 5. Analyze the effect of higher codimension on the regularity in Theorem 7.1 and Corollary 7.2

In other words, interpolate between Theorem 7.1 (codimension 1) and Källen's result (codimension sufficiently large).

Regarding Onsager's conjecture the next important question is how to modify convex integration so that the weak solutions in Theorem 2.4 are Hölder continuous. 
Problem 6. Construct continuous and Hölder continuous solutions of incompressible Euler.

An explicit construction could lead to insights concerning several assumptions usually made in the statistical theory of turbulence (such as the locality of interactions between eddies, cf. [19, or the very idea of an energy cascade). It would also open the way toward proving a true homotopy-principle for weak solutions, in the long run perhaps shedding light on the global topology of the moduli space of solutions. A first result in this direction has been recently obtained in 33.

Problem 7. Calculate/estimate the maximal dissipation rate for wild initial data.

By a simple contradiction argument it can be seen that there has to be a finite maximal rate. A first example might be the shear flow, cf. with Theorem 3.4. For the very similar problem of the incompressible porous medium equation (see Theorem 4.7), the maximal expansion rate of the mixing zone has been obtained in 65.

Apart from a dissipation rate principle, the natural candidate for a selection principle for the incompressible Euler equations is the vanishing viscosity limit. Of course this has several major technical stumbling blocks, but already a "recovery sequence" in the vanishing viscosity limit would be of interest:

Problem 8. Recovery sequence for weak solutions of Euler from Navier-Stokes.

It is conceivable that one would need to consider Navier-Stokes with a random perturbation. Indeed, adding noise is not just physically motivated, but in some sense necessary: without noise the statement is false (see Theorem 3.4), at least for Leray solutions. On the other hand, it is not excluded that a suitable version of the $h$-principle holds for weaker notions of solutions, e.g. mild solutions.

Problem 9. Probabilistic approach to convex integration.

Convex integration can be seen as a control problem: at each step of the iteration, one has to choose an admissible perturbation, consisting essentially of a (plane-)wave direction and a frequency. The following is therefore a natural question: are there meaningful optimality conditions?

Finally, up to now there has been no attempt to link weak solutions and/or subsolutions to generalized flows or to Brenier's sharp measure-valued solution [13]:

Problem 10. Relation of subsolutions to generalized flows.

The general philosophy of convex integration is to exploit the lack of weak compactness (compare with the discussion in Section 4). For certain problems such as for active scalar equations with an odd multiplier (for instance SQG) or twodimensional Euler in the Delort class [34, the solution space is weakly (but not strongly) compact [25, 69]. This seems to obstruct the applicability of convex integration, but leaves the question of uniqueness open.

Problem 11. The uniqueness or nonuniqueness for SQG.

\section{ACKNOWLEDGMENTS}

The authors are grateful to Emil Wiedemann for several comments on earlier versions of the paper. The first author acknowledges the support of the SFB Grant TR71, the second author acknowledges the support of the ERC Grant Agreement No. 277993 and the support of the Hausdorff Center for Mathematics in Bonn. 


\section{ABOUT THE AUTHORS}

Camillo De Lellis graduated in 2002 at the Scuola Normale Superiore di Pisa under the supervision of Luigi Ambrosio and he is currently professor at the University of Zürich. He has received the Stampacchia medal in 2009. He works in partial differential equations and geometric measure theory.

László Székelyhidi graduated in 2003 at the Max Planck Institute for Mathematics in the Sciences under the supervision of Stefan Müller, and he is currently professor at the University of Leipzig. He has been awarded the Oberwolfach prize in 2010. His main interests are in partial differential equations and calculus of variations.

\section{REFERENCES}

1. J. J. Alibert and G. Bouchitté, Non-uniform integrability and generalized young measure, Journal of Convex Analysis 4 (1997), no. 1, 129-147. MR1459885 (99i:28007)

2. C. Bardos, Jean-Michel Ghidaglia, and Spyridon Kamvissis, Weak convergence and deterministic approach to turbulent diffusion, Nonlinear wave equations (Providence, RI, 1998), Contemp. Math., vol. 263, Amer. Math. Soc., 2000, pp. 1-15. MR1777632 (2001e:76076)

3. K. Bardos and È. S. Titi, Euler equations for an ideal incompressible fluid, Uspekhi Mat. Nauk 62 (2007), no. 3(375), 5-46. MR2355417 (2008f:76017)

4. J. T. Beale, T. Kato, and A. Majda, Remarks on the breakdown of smooth solutions for the 3-D Euler equations, Comm. Math. Phys. 94 (1984), no. 1, 61-66. MR763762 (85j:35154)

5. Michael Blaser and Tristan Rivière, A minimality property for entropic solutions to scalar conservation laws in $1+1$ dimensions, Comm. Partial Differential Equations 35 (2010), no. 10, 1763-1801. MR2754068

6. Ju. F. Borisov, The parallel translation on a smooth surface. I, Vestnik Leningrad. Univ. 13 (1958), no. 7, 160-171. MR0104277(21:3032)

7. no. 19, 45-54. MR0104278 (21:3033)

8. _ On the connection bewteen the spatial form of smooth surfaces and their intrinsic geometry, Vestnik Leningrad. Univ. 14 (1959), no. 13, 20-26. MR0116295 (22:7090)

9. __ The parallel translation on a smooth surface. III, Vestnik Leningrad. Univ. 14 (1959), no. 1, 34-50. MR0104279 (21:3034)

10. On the question of parallel displacement on a smooth surface and the connection of space forms of smooth surfaces with their intrinsic geometries., Vestnik Leningrad. Univ. 15 (1960), no. 19, 127-129. MR0131225 (24:A1078)

11.,$C^{1, \alpha}$-isometric immersions of Riemannian spaces, Doklady 163 (1965), 869-871.

12. Yu.F. Borisov, Irregular $C^{1, \beta}$-surfaces with analytic metric., Sib. Mat. Zh. 45 (2004), no. 1, 25-61 (Russian, English). MR2047871 (2005a:53113)

13. Yann Brenier, Minimal geodesics on groups of volume-preserving maps and generalized solutions of the Euler equations, Comm. Pure Appl. Math. 52 (1999), no. 4, 411-452. MR.1658919 (2000c:58020)

14. Convergence of the Vlasov-Poisson system to the incompressible Euler equations, Comm. in Partial Differential Equations 25 (2000), no. 3, 737-754. MR1748352 (2001c:76124)

15. Yann Brenier, Camillo De Lellis, and László Székelyhidi, Jr., Weak-strong uniqueness for measure valued solutions, Comm. Math. Phys. 305 (2011), no. 2, 351-361. MR2805464

16. Yann Brenier and Emmanuel Grenier, Limite singulière du système de Vlasov-Poisson dans le régime de quasi neutralité: le cas indépendant du temps, C. R. Acad. Sci. Paris Sér. I Math. 318 (1994), no. 2, 121-124. MR1260322 (95b:82057)

17. Alberto Bressan and Fabián Flores, On total differential inclusions, Rend. Sem. Mat. Univ. Padova 92 (1994), 9-16. MR1320474 (96b:35244)

18. Arrigo Cellina, On the differential inclusion $x^{\prime} \in[-1,+1]$, Atti Accad. Naz. Lincei Rend. Cl. Sci. Fis. Mat. Natur. (8) 69 (1980), no. 1-2, 1-6 (1981). MR641583 (83g:34012)

19. A. Cheskidov, P. Constantin, S. Friedlander, and R. Shvydkoy, Energy conservation and Onsager's conjecture for the Euler equations, Nonlinearity 21 (2008), no. 6, 1233-1252. MR2422377 (2009g:76008) 
20. E. Chiodaroli, A counterexample to well-posedness of entropy solutions to the compressible Euler system, Preprint (2012).

21. Alexandre J. Chorin, Vorticity and turbulence, Applied Mathematical Sciences, vol. 103, Springer-Verlag, New York, 1994. MR1281384 (95m:76043)

22. St. Cohn-Vossen, Zwei Sätze über die Starrheit der Eiflächen., Nachrichten Göttingen 1927 (1927), 125-137 (German).

23. P. Constantin, On the Euler equations of incompressible fluids, Bull. Amer. Math. Soc. (N.S.) 44 (2007), no. 4, 603-621. MR2338368(2008i:76010)

24. Peter Constantin, Weinan E, and Edriss S. Titi, Onsager's conjecture on the energy conservation for solutions of Euler's equation, Comm. Math. Phys. 165 (1994), no. 1, 207-209. MR.1298949 (96e:76025)

25. Peter Constantin, Andrew J. Majda, and Esteban Tabak, Formation of strong fronts in the 2D quasigeostrophic thermal active scalar, Nonlinearity 7 (1994), no. 6, 1495-1533. MR 1304437 (95i:76107)

26. Sergio Conti, Camillo De Lellis, and László Székelyhidi, Jr., h-principle and rigidity for $C^{1, \alpha}$ isometric embeddings, Nonlinear Partial Differential Equations, The Abel Symposium 2010 (Helge Holden and Kenneth H. Karlsen, eds.), Springer-Verlag, 2012, pp. 83-116.

27. Diego Cordoba, Daniel Faraco, and Francisco Gancedo, Lack of uniqueness for weak solutions of the incompressible porous media equation, Arch. Ration. Mech. Anal. 200 (2011), no. 3, 725-746. MR2796131

28. Bernard Dacorogna and Paolo Marcellini, General existence theorems for Hamilton-Jacobi equations in the scalar and vectorial cases, Acta Math. 178 (1997), 1-37. MR98d:35029

29. Constantine M. Dafermos, The entropy rate admissibility criterion for solutions of hyperbolic conservation laws, J. Differential Equations 14 (1973), 202-212. MR0328368(48:6710)

30. Hyperbolic conservation laws in continuum physics, Grundlehren der Mathematischen Wissenschaften [Fundamental Principles of Mathematical Sciences], vol. 325, Springer-Verlag, Berlin, 2000. MR:1763936 (2001m:35212)

31. Camillo De Lellis and László Székelyhidi, Jr., The Euler equations as a differential inclusion, Ann. of Math. (2) 170 (2009), no. 3, 1417-1436. MR2600877(2011e:35287)

32. - On admissibility criteria for weak solutions of the Euler equations, Arch. Ration. Mech. Anal. 195 (2010), no. 1, 225-260. MR2564474 (2011d:35386)

33. Continuous dissipative Euler flows, Preprint. (2012).

34. Jean-Marc Delort, Existence de nappes de tourbillon en dimension deux, J. Amer. Math. Soc. 4 (1991), no. 3, 553-586. MR 1102579 (92f:76019)

35. Ronald J. DiPerna, Compensated compactness and general systems of conservation laws, Trans. Amer. Math. Soc. 292 (1985), no. 2, 383-420. MR808729 (87g:35148)

36. Ronald J. DiPerna and Andrew J. Majda, Oscillations and concentrations in weak solutions of the incompressible fluid equations, Comm. Math. Phys. 108 (1987), no. 4, 667-689. MR877643 (88a:35187)

37. Jean Duchon and Raoul Robert, Inertial energy dissipation for weak solutions of incompressible Euler and Navier-Stokes equations, Nonlinearity 13 (2000), no. 1, 249-255. MR.1734632 (2001c:76032)

38. David G. Ebin and Jerrold Marsden, Groups of diffeomorphisms and the motion of an incompressible fluid., Ann. of Math. (2) 92 (1970), 102-163. MR0271984 (42:6865)

39. Y. Eliashberg and N. Mishachev, Introduction to the h-principle, Graduate Studies in Mathematics, vol. 48, American Mathematical Society, Providence, RI, 2002. MR.1909245 (2003g:53164)

40. Gregory L. Eyink, Energy dissipation without viscosity in ideal hydrodynamics. I. Fourier analysis and local energy transfer, Phys. D 78 (1994), no. 3-4, 222-240. MR.1302409 (95m:76020)

41. Gregory L. Eyink and Katepalli R. Sreenivasan, Onsager and the theory of hydrodynamic turbulence, Reviews of Modern Physics 78 (2006). MR2214822 (2007g:76108)

42. C. L. Fefferman, Existence and smoothness of the Navier-Stokes equation, The Millennium Prize Problems, Clay Math. Inst., Cambridge, MA, 2006, pp. 57-67. MR2238274

43. Mikhael Gromov, Partial differential relations, Ergebnisse der Mathematik und ihrer Grenzgebiete (3), vol. 9, Springer-Verlag, Berlin, 1986. MR90a:58201

44. __ Local and global in geometry, Balzan Prize, 1999. 
45. G. Herglotz, Über die Starrheit der Eiflächen., Abh. Math. Semin. Hansische Univ. 15 (1943), 127-129 (German). MR0014714 (7:322a)

46. Anders Källén, Isometric embedding of a smooth compact manifold with a metric of low regularity, Ark. Mat. 16 (1978), no. 1, 29-50. MR499136 (80c:53064)

47. Tosio Kato, Nonstationary flows of viscous and ideal fluids in $\mathbf{R}^{3}$, J. Functional Analysis 9 (1972), 296-305. MR0481652 (58:1753)

48. B. Kirchheim, Rigidity and Geometry of microstructures, Habilitation thesis, University of Leipzig, 2003.

49. Bernd Kirchheim, Stefan Müller, and Vladimir Šverák, Studying nonlinear PDE by geometry in matrix space, Geometric Analysis and Nonlinear Partial Differential Equations (Stefan Hildebrandt and Hermann Karcher, eds.), Springer-Verlag, 2003, pp. 347-395. MR2008346 (2006f:35087)

50. Herbert Koch, Transport and instability for perfect fluids, Math. Ann. 323 (2002), no. 3, 491-523. MR1923695 (2003f:76009)

51. N. Kuiper, On $C^{1}$ isometric imbeddings i,ii, Proc. Kon. Acad. Wet. Amsterdam A 58 (1955), 545-556, 683-689. MR0075640 (17:782c)

52. P. D. Lax, Deterministic theories of turbulence, Frontiers in Pure and Applied Mathematics, North-Holland, Amsterdam, 1991, pp. 179-184. MR1110599 (92i:76058)

53. Leon Lichtenstein, Grundlagen der Hydromechanik, Die Grundlehren der Mathematischen Wissenschaften in Einzeldarstellungen, Band 30, Springer-Verlag, Berlin, 1968. MR0228225 $(37: 3808)$

54. Pierre-Louis Lions, Mathematical topics in fluid mechanics. Vol. 1, Oxford Lecture Series in Mathematics and its Applications, vol. 3, The Clarendon Press Oxford University Press, New York, 1996, Incompressible Models, Oxford Science Publications. MR,1422251 (98b:76001)

55. Andrew J. Majda and Andrea L. Bertozzi, Vorticity and incompressible flow, Cambridge Texts in Applied Mathematics, vol. 27, Cambridge University Press, Cambridge, 2002. MR.1867882 (2003a:76002)

56. Carlo Marchioro and Mario Pulvirenti, Mathematical theory of incompressible nonviscous fluids, Applied Mathematical Sciences, vol. 96, Springer-Verlag, New York, 1994. MR.1245492 (94k:76001)

57. J. Moser, A rapidly convergent iteration method and non-linear partial differential equations I, Ann. Scuola Norm. Sup. Pisa 20 (1966), 265-315. MR0199523 (33:7667)

58. _ A rapidly convergent iteration method and non-linear partial differential equations II, Ann. Scuola Norm. Sup. Pisa 20 (1966), 499-535. MR0206461 (34:6280)

59. S. Müller and V. Šverák, Convex integration for Lipschitz mappings and counterexamples to regularity, Ann. of Math. (2) 157 (2003), no. 3, 715-742. MR.1983780 (2005i:35028)

60. Stefan Müller, Variational models for microstructure and phase transitions, Calculus of Variations and Geometric Evolution Problems (Cetraro, 1996), Lecture Notes in Math., vol. 1713, Springer, Berlin, 1999, pp. 85-210. MR.1731640 (2001b:49019)

61. J. Nash, $C^{1}$ isometric imbeddings, Ann. Math. 60 (1954), 383-396. MR0065993 (16:515e)

62. __ The imbedding problem for Riemannian manifolds, Ann. Math. 63 (1956), 20-63. MR0075639(17:782b)

63. L. Onsager, Statistical hydrodynamics, Nuovo Cimento (9) 6 (1949), no. Supplemento, 2(Convegno Internazionale di Meccanica Statistica), 279-287. MR0036116 (12,60f)

64. Felix Otto, Evolution of microstructure in unstable porous media flow: a relaxational approach, Comm. Pure Appl. Math. 52 (1999), no. 7, 873-915. MR1682800 (2000i:76120)

65. __ Evolution of microstructure: an example, Ergodic Theory, Analysis, and Efficient Simulation of Dynamical Systems, Springer, Berlin, 2001, pp. 501-522. MR.1850320 (2002f:76036)

66. Pablo Pedregal, Laminates and microstructure, European J. Appl. Math. 4 (1993), 121-149. MR.95g:73035

67. A. V. Pogorelov, Extrinsic geometry of convex surfaces, American Mathematical Society, Providence, R.I., 1973, Translations of Mathematical Monographs, Vol. 35. MR.0346714 (49:11439)

68. A. V. Pogorelov, The rigidity of general convex surfaces, Doklady Acad. Nauk SSSR 79 (1951), 739-742. MR0043488 (13:271b)

69. S. Resnick, Dynamical problems in nonlinear advective partial differential equations, Ph.D. thesis, University of Chicago, 1995. 
70. I. H. Sabitov, Regularity of convex domains with a metric that is regular on Hölder classes, Sibirsk. Mat. Ž. 17 (1976), no. 4, 907-915. MR0425854 (54:13804)

71. Vladimir Scheffer, An inviscid flow with compact support in space-time, J. Geom. Anal. 3 (1993), no. 4, 343-401. MR1231007 (94h:35215)

72. Denis Serre, Systems of conservation laws. 1, Cambridge University Press, Cambridge, 1999, Hyperbolicity, entropies, shock waves, Translated from the 1996 French original by I. N. Sneddon. MR 1707279 (2000g:35142)

73. A. Shnirelman, On the nonuniqueness of weak solution of the Euler equation, Comm. Pure Appl. Math. 50 (1997), no. 12, 1261-1286. MR1476315 (98j:35149)

74. _ Weak solutions with decreasing energy of incompressible Euler equations, Comm. Math. Phys. 210 (2000), no. 3, 541-603. MR1777341 (2002g:76009)

75. R. Shvydkoy, Convex integration for a class of active scalar equations, J. Amer. Math. Soc. 24 (2011), no. 4, 1159-1174. MR2813340

76. Roman Shvydkoy, Lectures on the Onsager conjecture, Discrete Contin. Dyn. Syst. Ser. S 3 (2010), no. 3, 473-496. MR2660721(2011h:76051)

77. Michael Spivak, A comprehensive introduction to differential geometry. Vol. V. 2nd ed., Berkeley: Publish or Perish, Inc., 1979 (English). MR.532834 (82g:53003e)

78. David Spring, On the regularity of solutions in convex integration theory, Invent. Math. 104 (1991), no. 1, 165-178. MR1094050 (92e:35044)

79. M. A. Sychev, A few remarks on differential inclusions, Proc. Roy. Soc. Edinburgh Sect. A 136 (2006), no. 3, 649-668. MR2227812 (2006m:34027)

80. László Székelyhidi, Jr., Weak solutions to the incompressible Euler equations with vortex sheet initial data, C. R. Acad. Sci. Paris Sér. I Math. 349 (2011), no. 19-20, 1063-1066. MR.2842999

81. Relaxation of the incompressible porous medium equation, to appear in Ann. Sci. Ec. Norm. Sup. (2012).

82. László Székelyhidi, Jr. and E. Wiedemann, Young measures generated by ideal incompressible fluid flows, to appear in Arch. Rat. Mech. Anal. (2012).

83. Luc Tartar, Compensated compactness and applications to partial differential equations, Nonlinear analysis and mechanics: Heriot-Watt Symposium, Vol. IV, Res. Notes in Math., vol. 39, Pitman, Boston, Mass., 1979, pp. 136-212. MR584398 (81m:35014)

84. ㄴ The compensated compactness method applied to systems of conservation laws, Systems of Nonlinear Partial Differential Equations (Oxford, 1982), NATO Adv. Sci. Inst. Ser. C Math. Phys. Sci., vol. 111, Reidel, Dordrecht, 1983, pp. 263-285. MR725524 (85e:35079)

85. Emil Wiedemann, Existence of weak solutions for the incompressible Euler equations, Ann. Inst. H. Poincaré Anal. Non Linéaire 28 (2011), no. 5, 727-730. MR2838398

86. Shing-Tung Yau, Open problems in geometry, Differential Geometry: Partial Differential Equations on Manifolds (Los Angeles, CA, 1990), Proc. Sympos. Pure Math., vol. 54, Amer. Math. Soc., Providence, RI, 1993, pp. 1-28. MR1216573 (94k:53001)

Institut Für MAThematik, Universität ZürICH, CH-8057 ZüRICH

E-mail address: camillo.delellis@math.unizh.ch

Institut Für MAThematik, Universität LeIPZIG, D-04103 LEIPZIG

E-mail address: laszlo.szekelyhidi@math.uni-leipzig.de 